ITP-UU-12/47

SPIN-12/44

\title{
Scalar product of Bethe vectors from functional equations
}

\author{
W. Galleas \\ Institute for Theoretical Physics and Spinoza Institute, \\ Utrecht University, Leuvenlaan 4, 3584 CE Utrecht, \\ The Netherlands \\ w.galleas@uu.nl
}

\begin{abstract}
In this work the scalar product of Bethe vectors for the six-vertex model is studied by means of functional equations. The scalar products are shown to obey a system of functional equations originated from the Yang-Baxter algebra and its solution is given as a multiple contour integral.
\end{abstract}

PACS numbers: $05.50+\mathrm{q}, 02.30 . \mathrm{IK}$

Keywords: Six-vertex model, functional equations, scalar product 


\section{Contents}

1 Introduction $\quad 2$

2 Bethe vectors for the six-vertex model $\quad 3$

$\begin{array}{lll}3 & \text { Functional equations } & 6\end{array}$

3.1 Equation type A . . . . . . . . . . . . . . . . 6

3.2 Equation type D . . . . . . . . . . . . . . . 7

$\begin{array}{llr}4 & \text { Scalar product } & 8\end{array}$

4.1 Off-shell formula . . . . . . . . . . . . . . . . . . . . 10

4.2 On-shell formula . . . . . . . . . . . . . . . . . 17

5 Concluding remarks $\quad 19$

6 Acknowledgements $\quad 19$

$\begin{array}{lr}\text { A Polynomial structure } & 20\end{array}$

B Special zeroes $\quad 21$

B.1 Case $n=2 \ldots \ldots \ldots \ldots \ldots \ldots \ldots$

B.2 Case $n=3 \ldots \ldots \ldots \ldots \ldots \ldots \ldots$

B.3 General case . . . . . . . . . . . . . . . . . . . . 24

$\begin{array}{lll}\text { C } S_{n} \text { as a doubly symmetric function } & 24\end{array}$

$\begin{array}{ll}\text { D Asymptotic behaviour } & 26\end{array}$

$\begin{array}{ll}\text { E Off-shell solution for the case } n=1 & 28\end{array}$

\section{Introduction}

Some important models of quantum field theory and condensed matter physics exhibit remarkable properties granting them the gift of integrability. Among prominent examples we have the quantum non-linear Schrödinger model [1], the Heisenberg spin chain [2] and the Hubbard model $[3]$. The realm of those models are of quantum nature and, although a rigorous and unambiguous definition of integrability at quantum level is still lacking [4-7], these models exhibit a set of enhanced symmetries which can be explored in order to compute physical quantities exactly $[8,9]$. Among those quantities we mention the energy spectrum and some correlation functions, and in this way integrability offers nonperturbative access to the behaviour of strongly interacting systems. These achievements are in large part due to the advent of the Bethe ansatz $[10]$ and its algebraic formulation $[11,12]$ characterising the model wave function in terms of its momentum. This algebraic formulation has been successfully applied to the majority of known integrable systems [13-16], yielding the model eigenvectors in terms of creation operators acting on a suitable 
reference state. Moreover, as remarked in [17] the study of Bethe's wave function also provided important insights leading to the concept of commuting transfer matrices [18].

On the other hand, the exact solution of a model whose transfer matrix belongs to a commutative family is also intimately connected with functional equations methods [19]. As far as spectral properties are concerned, we have available a variety of functional methods $\left[\begin{array}{ll}19 & 22\end{array}\right.$ yielding transfer matrices eigenvalues which do not address the problem of explicitly constructing the respective eigenvectors. In fact, the evaluation of most physical quantities do not require the eigenvectors themselves but quantities which can be derived from them. For instance, the calculation of correlation functions would then require the evaluation of eigenvectors scalar products in addition to the expected value of operators [8].

Within the framework of the algebraic Bethe ansatz, the calculation of scalar product of Bethe vectors was inaugurated by Korepin [23] largely influenced by Gaudin's hypothesis on the norms of the non-linear Schrödinger model wave function [24]. The method of [23] has also been described in [8] where the scalar product of Bethe vectors for the six-vertex model is given as the vacuum mean value of a determinant whose entries are expressed in terms of quantum fields. In $[8,25$ that scalar product is also given as a summation over a product of determinants with scalar entries.

The purpose of this paper is to demonstrate that scalar product of Bethe vectors can also be computed by means of functional equations. The method we shall employ here resembles the one described in the series of works $[26-29]$ where the Yang-Baxter algebra has played the major role in deriving functional equations describing the partition functions of vertex and SOS models with domain wall boundaries. The origin of the functional equations describing scalar products is also the Yang-Baxter algebra and its solution turns out to be given by a multiple contour integral.

This paper is organised as follows. In Section 2 we give a brief description of Bethe vectors for the six-vertex model. In Section 3 we illustrate how the Yang-Baxter algebra can be explored in order to derive functional equations describing scalar products. The solution of our functional equations is obtained in Section 4 and concluding remarks are discussed in Section 5. Technical details and proofs are presented through the Appendices A to E.

\section{Bethe vectors for the six-vertex model}

Vertex models in two dimensions constitute one of the corner stones of the theory of exactly solvable models of statistical mechanics [30]. In particular, the study of the eigenvectors of the six-vertex model $[31]$ by means of the Bethe ansatz [10] provided insightful information which paved the way to establish the connection between twodimensional vertex models and one-dimensional spin chains [32,33, 18]. This study also received a large impulse with the advent of the Quantum Inverse Scattering Method (QISM) which unveiled the algebraic foundation supporting the construction of Bethe vectors $[11,12]$. 
Bethe vectors. In the framework of the QISM, the eigenvectors of the six-vertex model are built up from the action of creation operators on a pseudo-vacuum state. More precisely, an eigenvector $|\psi\rangle$ is of the form

$$
|\psi\rangle=B\left(\lambda_{1}^{B}\right) \ldots B\left(\lambda_{n}^{B}\right)|0\rangle
$$

where $B(\lambda)$, together with three more generators $A(\lambda), C(\lambda)$ and $D(\lambda)$, satisfy a certain set of algebraic relations. In its turn, the vector $|0\rangle$ is the $\mathfrak{s l}_{2}$ highest weight vector while the parameters $\lambda_{j}^{B} \in \mathbb{C}$ are required to satisfy certain constraints.

Yang-Baxter algebra. The algebra satisfied by the generators $A(\lambda), B(\lambda), C(\lambda)$ and $D(\lambda)$ is commonly referred to as Yang-Baxter algebra and it reads

$$
\mathcal{R}_{12}\left(\lambda_{1}-\lambda_{2}\right) \mathcal{T}_{1}\left(\lambda_{1}\right) \mathcal{T}_{2}\left(\lambda_{2}\right)=\mathcal{T}_{2}\left(\lambda_{2}\right) \mathcal{T}_{1}\left(\lambda_{1}\right) \mathcal{R}_{12}\left(\lambda_{1}-\lambda_{2}\right)
$$

The relation $(2.2)$ is defined in $\operatorname{End}\left(\mathbb{V}_{1} \otimes \mathbb{V}_{2}\right)$ with $\mathbb{V}_{j} \cong \mathbb{C}^{2}$. In their turn, $\mathcal{T}_{1}=\mathcal{T} \otimes$ id and $\mathcal{T}_{2}=\mathrm{id} \otimes \mathcal{T}$ with

$$
\mathcal{T}(\lambda)=\left(\begin{array}{ll}
A(\lambda) & B(\lambda) \\
C(\lambda) & D(\lambda)
\end{array}\right)
$$

The matrix $\mathcal{R}_{a b} \in \operatorname{End}\left(\mathbb{V}_{a} \otimes \mathbb{V}_{b}\right)$ encodes the algebra structure constants associated with the six-vertex model and it is explicitly given by

$$
\mathcal{R}=\left(\begin{array}{llll}
a & 0 & 0 & 0 \\
0 & b & c & 0 \\
0 & c & b & 0 \\
0 & 0 & 0 & a
\end{array}\right)
$$

where $a(\lambda)=\sinh (\lambda+\gamma), b(\lambda)=\sinh (\lambda)$ and $c(\lambda)=\sinh (\gamma)$. The $\mathcal{R}$-matrix $(2.4)$ satisfies the Yang-Baxter equation

$$
\mathcal{R}_{12}\left(\lambda_{1}-\lambda_{2}\right) \mathcal{R}_{13}\left(\lambda_{1}-\lambda_{3}\right) \mathcal{R}_{23}\left(\lambda_{2}-\lambda_{3}\right)=\mathcal{R}_{23}\left(\lambda_{2}-\lambda_{3}\right) \mathcal{R}_{13}\left(\lambda_{1}-\lambda_{3}\right) \mathcal{R}_{12}\left(\lambda_{1}-\lambda_{2}\right)
$$

in $\operatorname{End}\left(\mathbb{V}_{1} \otimes \mathbb{V}_{2} \otimes \mathbb{V}_{3}\right)$ ensuring the associativity of $(2.2)$.

Representations. The representations of $A(\lambda), B(\lambda), C(\lambda)$ and $D(\lambda)$ in the tensor product space $\mathbb{V}_{1} \otimes \cdots \otimes \mathbb{V}_{L}$ can be built as follows. We consider an ordered product of $\mathcal{R}$-matrices in the tensor product space $\mathbb{V}_{a} \otimes \mathbb{V}_{1} \otimes \cdots \otimes \mathbb{V}_{L}$, namely

$$
\overline{\mathcal{T}}_{a}(\lambda)=\overrightarrow{\prod_{1 \leq j \leq L}} \mathcal{R}_{a j}\left(\lambda-\mu_{j}\right),
$$

with parameters $\lambda, \mu_{j} \in \mathbb{C}$. Due to $(2.5)$ one can show that $\overline{\mathcal{T}}_{a}$ satisfy $(2.2)$ and thus $\mathcal{T}=\overline{\mathcal{T}}_{a}$ yields a representation of $A(\lambda), B(\lambda), C(\lambda)$ and $D(\lambda)$. 
Highest weight vector. The vector $|0\rangle$ in $(2.1)$ is the $\mathfrak{s l}_{2}$ highest weight vector and it explicitly reads

$$
|0\rangle=\bigotimes_{j=1}^{L}\left(\begin{array}{l}
1 \\
0
\end{array}\right)
$$

in the space $\mathbb{V}_{1} \otimes \cdots \otimes \mathbb{V}_{L}$. Taking into consideration the representation (2.6) with $\mathcal{R}$-matrix given by $(2.4)$, we readily obtain the properties

$$
\begin{aligned}
A(\lambda)|0\rangle & =\prod_{j=1}^{L} a\left(\lambda-\mu_{j}\right)|0\rangle & \langle 0| A(\lambda) & =\prod_{j=1}^{L} a\left(\lambda-\mu_{j}\right)\langle 0| \\
D(\lambda)|0\rangle & =\prod_{j=1}^{L} b\left(\lambda-\mu_{j}\right)|0\rangle & \langle 0| D(\lambda) & =\prod_{j=1}^{L} b\left(\lambda-\mu_{j}\right)\langle 0| \\
C(\lambda)|0\rangle & =0 & \langle 0| B(\lambda) & =0,
\end{aligned}
$$

where $\langle 0|$ corresponds to the transposition of $|0\rangle$.

Twisted transfer matrix. The Yang-Baxter algebra (2.2) enables us to show that the matrix $T(\lambda)=\phi_{1} A(\lambda)+\phi_{2} D(\lambda)$ forms an one-parameter family of mutually commuting matrices, i.e. $\left[T\left(\lambda_{1}\right), T\left(\lambda_{2}\right)\right]=0$. The matrix $T(\lambda)$ is called twisted transfer matrix and for vertex models it represents the configuration of rows spanning a two-dimensional lattice with periodic boundary conditions. In their turn, the parameters $\phi_{1}, \phi_{2} \in \mathbb{C}$ govern the deviations from strict toroidal boundary conditions and, in this sense, they introduce the notion of twisted boundary conditions [34]. The vector $|\psi\rangle$ as defined by $(2.1)$ is an eigenvector of $T(\lambda)$ with eigenvalue

$$
\Lambda(\lambda)=\phi_{1} \prod_{j=1}^{L} a\left(\lambda-\mu_{j}\right) \prod_{i=1}^{n} \frac{a\left(\lambda_{i}^{B}-\lambda\right)}{b\left(\lambda_{i}^{B}-\lambda\right)}+\phi_{2} \prod_{j=1}^{L} b\left(\lambda-\mu_{j}\right) \prod_{i=1}^{n} \frac{a\left(\lambda-\lambda_{i}^{B}\right)}{b\left(\lambda-\lambda_{i}^{B}\right)}
$$

for particular choices of the parameters $\lambda_{i}^{B}$.

Dual Bethe vector. The dual vector

$$
\langle\psi|=\langle 0| C\left(\lambda_{1}^{B}\right) \ldots C\left(\lambda_{n}^{B}\right)
$$

is an eigenvector of $T$, i.e. $\langle\psi| T(\lambda)=\Lambda(\lambda)\langle\psi|$ with the same eigenvalue $\Lambda$ as given by (2.9). Nevertheless, here we shall consider the dual vector

$$
\langle\widetilde{\psi}|=\langle 0| C\left(\lambda_{1}^{C}\right) \ldots C\left(\lambda_{n}^{C}\right)
$$

in order to keep our results as general as possible. 
Off-shell scalar product. The scalar product of Bethe vectors $S_{n}$ is then defined as

$$
S_{n}\left(\lambda_{1}^{C}, \ldots, \lambda_{n}^{C} \mid \lambda_{1}^{B}, \ldots, \lambda_{n}^{B}\right)=\left\langle 0\left|\prod_{i=1}^{n} C\left(\lambda_{i}^{C}\right) \prod_{i=1}^{n} B\left(\lambda_{i}^{B}\right)\right| 0\right\rangle .
$$

We shall refer to (2.12) as off-shell scalar product when the variables $\lambda_{i}^{B}$ and $\lambda_{i}^{C}$ are free to assume any value on the complex plane.

On-shell scalar product. The vector $|\psi\rangle$ defined in $(2.1)$ is an eigenvector of the twisted transfer matrix $T(\lambda)$ only when the variables $\lambda_{i}^{B}$ satisfy certain constraints. Thus the parameters $\lambda_{i}^{B}$ and $\lambda_{i}^{C}$ need to be fine tuned in order to having (2.12) describing the norm of a Bethe vector. The constraints on the variables $\lambda_{i}^{B}$ ensuring that $(2.1)$ is a transfer matrix eigenvector are the so called Bethe ansatz equations. Similar constraints would be required for the dual eigenvector $(2.11)$ but here we shall consider a less restrictive condition by keeping the variables $\lambda_{i}^{C}$ arbitrary. In this way the scalar product $(2.12)$ under the condition

$$
\prod_{j=1}^{L} \frac{a\left(\lambda_{i}^{B}-\mu_{j}\right)}{b\left(\lambda_{i}^{B}-\mu_{j}\right)}=(-1)^{n-1} \frac{\phi_{2}}{\phi_{1}} \prod_{\substack{k=1 \\ k \neq i}}^{n} \frac{a\left(\lambda_{i}^{B}-\lambda_{k}^{B}\right)}{a\left(\lambda_{k}^{B}-\lambda_{i}^{B}\right)}
$$

will be referred to as on-shell scalar product.

\section{$3 \quad$ Functional equations}

This section is devoted to the derivation of functional equations describing the scalar product $S_{n}$ defined in (2.12). The main ingredient of our derivation is the commutation rules encoded in the relation (2.2) commonly referred to as Yang-Baxter algebra. We shall obtain two different functional equations and the determination of $S_{n}$ will rely on the resolution of this system of equations. This method consists of an extension of the one originally proposed in [26] for the partition function of the six-vertex model with domain wall boundaries and subsequently generalised in $[28,29]$ for SOS models. Similarly to those cases, the derivation of functional equations for scalar products will explore a consistency relation between the $\mathfrak{s l}_{2}$ algebra highest weight representation theory and the Yang-Baxter algebra.

\subsection{Equation type A}

We consider the quantity

$$
\left\langle 0\left|\prod_{i=1}^{n} C\left(\lambda_{j}^{C}\right) A\left(\lambda_{0}\right) \prod_{i=1}^{n} B\left(\lambda_{j}^{B}\right)\right| 0\right\rangle
$$


computed in two different ways. In the first way we consider the commutation rules

$$
\begin{aligned}
B\left(\lambda_{1}\right) B\left(\lambda_{2}\right) & =B\left(\lambda_{2}\right) B\left(\lambda_{1}\right) \\
A\left(\lambda_{1}\right) B\left(\lambda_{2}\right) & =\frac{a\left(\lambda_{2}-\lambda_{1}\right)}{b\left(\lambda_{2}-\lambda_{1}\right)} B\left(\lambda_{2}\right) A\left(\lambda_{1}\right)-\frac{c\left(\lambda_{2}-\lambda_{1}\right)}{b\left(\lambda_{2}-\lambda_{1}\right)} B\left(\lambda_{1}\right) A\left(\lambda_{2}\right)
\end{aligned}
$$

contained in the relation $(2.2)$ to move the operator $A\left(\lambda_{0}\right)$ in $(3.1)$ to the right through the string of operators $B\left(\lambda_{j}^{B}\right)$. Due to $(3.2)$, at the last step we shall need to compute the action of a given operator $A(\lambda)$ on the state $|0\rangle$. For that we use the relation $(2.8)$ arising from the $\mathfrak{s l}_{2}$ highest weight representation theory. By doing so we find that the quantity (3.1) consists of a linear combination of terms $S_{n}$ with $B$-type arguments in the set $\left\{\lambda_{0}, \lambda_{1}^{B}, \ldots, \lambda_{n}^{B}\right\}$ with only $n$ elements being taken at a time.

The second way of evaluating (3.1) is by moving the operator $A\left(\lambda_{0}\right)$ to the left through all the operators $C\left(\lambda_{j}^{C}\right)$. This can be implemented with the help of the relations

$$
\begin{aligned}
C\left(\lambda_{1}\right) C\left(\lambda_{2}\right) & =C\left(\lambda_{2}\right) C\left(\lambda_{1}\right) \\
C\left(\lambda_{1}\right) A\left(\lambda_{2}\right) & =\frac{a\left(\lambda_{1}-\lambda_{2}\right)}{b\left(\lambda_{1}-\lambda_{2}\right)} A\left(\lambda_{2}\right) C\left(\lambda_{1}\right)-\frac{c\left(\lambda_{1}-\lambda_{2}\right)}{b\left(\lambda_{1}-\lambda_{2}\right)} A\left(\lambda_{1}\right) C\left(\lambda_{2}\right),
\end{aligned}
$$

which are also among the ones encoded in $(2.2)$. Then at the last stage we will need the quantity $\langle 0| A(\lambda)$ which is given in (2.8). Thus this alternative route of computing (3.1) also yields a linear combination of terms $S_{n}$ but this time with $C$-type arguments in the set $\left\{\lambda_{0}, \lambda_{1}^{C}, \ldots, \lambda_{n}^{C}\right\}$ where only $n$ variables are taken at a time.

In this way the consistency between these two routes of computing $(\overline{3.1})$ implies the functional equation

$$
\begin{aligned}
& M_{0} S_{n}\left(\lambda_{1}^{C}, \ldots, \lambda_{n}^{C} \mid \lambda_{1}^{B}, \ldots, \lambda_{n}^{B}\right)+\sum_{i=1}^{n} N_{i}^{(B)} S_{n}\left(\lambda_{1}^{C}, \ldots, \lambda_{n}^{C} \mid \lambda_{0}, \lambda_{1}^{B}, \ldots, \lambda_{i-1}^{B}, \lambda_{i+1}^{B}, \ldots, \lambda_{n}^{B}\right) \\
& +\sum_{i=1}^{n} N_{i}^{(C)} S_{n}\left(\lambda_{0}, \lambda_{1}^{C}, \ldots, \lambda_{i-1}^{C}, \lambda_{i+1}^{C}, \ldots, \lambda_{n}^{C} \mid \lambda_{1}^{B}, \ldots, \lambda_{n}^{B}\right)=0,
\end{aligned}
$$

with coefficients

$$
\begin{aligned}
M_{0} & =\prod_{j=1}^{L} a\left(\lambda_{0}-\mu_{j}\right)\left[\prod_{i=1}^{n} \frac{a\left(\lambda_{i}^{C}-\lambda_{0}\right)}{b\left(\lambda_{i}^{C}-\lambda_{0}\right)}-\prod_{i=1}^{n} \frac{a\left(\lambda_{i}^{B}-\lambda_{0}\right)}{b\left(\lambda_{i}^{B}-\lambda_{0}\right)}\right] \\
N_{i}^{(B, C)} & =\alpha_{B, C} \frac{c\left(\lambda_{i}^{B, C}-\lambda_{0}\right)}{b\left(\lambda_{i}^{B, C}-\lambda_{0}\right)} \prod_{j=1}^{L} a\left(\lambda_{i}^{B, C}-\mu_{j}\right) \prod_{j \neq i}^{n} \frac{a\left(\lambda_{j}^{B, C}-\lambda_{i}^{B, C}\right)}{b\left(\lambda_{j}^{B, C}-\lambda_{i}^{B, C}\right)},
\end{aligned}
$$

where $\alpha_{B}=1$ and $\alpha_{C}=-1$.

\subsection{Equation type D}

The same mechanism employed in Section 3.1 can also be considered starting with the quantity

$$
\left\langle 0\left|\prod_{i=1}^{n} C\left(\lambda_{j}^{C}\right) D\left(\lambda_{0}\right) \prod_{i=1}^{n} B\left(\lambda_{j}^{B}\right)\right| 0\right\rangle
$$


instead of (3.1). In that case, moving the operator $D\left(\lambda_{0}\right)$ to the right through the string of operators $B\left(\lambda_{j}^{B}\right)$ will require the use of the Yang-Baxter algebra relation

$$
D\left(\lambda_{1}\right) B\left(\lambda_{2}\right)=\frac{a\left(\lambda_{1}-\lambda_{2}\right)}{b\left(\lambda_{1}-\lambda_{2}\right)} B\left(\lambda_{2}\right) D\left(\lambda_{1}\right)-\frac{c\left(\lambda_{1}-\lambda_{2}\right)}{b\left(\lambda_{1}-\lambda_{2}\right)} B\left(\lambda_{1}\right) D\left(\lambda_{2}\right) .
$$

On the other hand, the commutation rule

$$
C\left(\lambda_{1}\right) D\left(\lambda_{2}\right)=\frac{a\left(\lambda_{2}-\lambda_{1}\right)}{b\left(\lambda_{2}-\lambda_{1}\right)} D\left(\lambda_{2}\right) C\left(\lambda_{1}\right)-\frac{c\left(\lambda_{2}-\lambda_{1}\right)}{b\left(\lambda_{2}-\lambda_{1}\right)} D\left(\lambda_{1}\right) C\left(\lambda_{2}\right)
$$

will be required in order to move the operator $D\left(\lambda_{0}\right)$ to the left through all the operators $C\left(\lambda_{j}^{C}\right)$. Besides that we shall also consider the commutation rules $\left[B\left(\lambda_{1}\right), B\left(\lambda_{2}\right)\right]=$ $\left[C\left(\lambda_{1}\right), C\left(\lambda_{2}\right)\right]=0$ and the properties $(2.8)$.

Thus the consistency condition between these two different ways of computing (3.6) leave us with the following functional equation,

$$
\begin{aligned}
& \widetilde{M}_{0} S_{n}\left(\lambda_{1}^{C}, \ldots, \lambda_{n}^{C} \mid \lambda_{1}^{B}, \ldots, \lambda_{n}^{B}\right)+\sum_{i=1}^{n} \widetilde{N}_{i}^{(B)} S_{n}\left(\lambda_{1}^{C}, \ldots, \lambda_{n}^{C} \mid \lambda_{0}, \lambda_{1}^{B}, \ldots, \lambda_{i-1}^{B}, \lambda_{i+1}^{B}, \ldots, \lambda_{n}^{B}\right) \\
& +\sum_{i=1}^{n} \widetilde{N}_{i}^{(C)} S_{n}\left(\lambda_{0}, \lambda_{1}^{C}, \ldots, \lambda_{i-1}^{C}, \lambda_{i+1}^{C}, \ldots, \lambda_{n}^{C} \mid \lambda_{1}^{B}, \ldots, \lambda_{n}^{B}\right)=0
\end{aligned}
$$

whose coefficients are explicitly given by

$$
\begin{aligned}
\widetilde{M}_{0} & =\prod_{j=1}^{L} b\left(\lambda_{0}-\mu_{j}\right)\left[\prod_{i=1}^{n} \frac{a\left(\lambda_{0}-\lambda_{i}^{C}\right)}{b\left(\lambda_{0}-\lambda_{i}^{C}\right)}-\prod_{i=1}^{n} \frac{a\left(\lambda_{0}-\lambda_{i}^{B}\right)}{b\left(\lambda_{0}-\lambda_{i}^{B}\right)}\right] \\
\widetilde{N}_{i}^{(B, C)} & =\alpha_{B, C} \frac{c\left(\lambda_{0}-\lambda_{i}^{B, C}\right)}{b\left(\lambda_{0}-\lambda_{i}^{B, C}\right)} \prod_{j=1}^{L} b\left(\lambda_{i}^{B, C}-\mu_{j}\right) \prod_{j \neq i}^{n} \frac{a\left(\lambda_{i}^{B, C}-\lambda_{j}^{B, C}\right)}{b\left(\lambda_{i}^{B, C}-\lambda_{j}^{B, C}\right)} .
\end{aligned}
$$

In summary, we have demonstrated in this section how the Yang-Baxter algebra can be explored in order to derive functional equations for the scalar product of Bethe vectors. We have obtained two distinct equations which we shall refer to as equation of type A (3.4) and equation of type D (3.9). The solution of these equations will be discussed in the next section.

\section{Scalar product}

Solving the system of functional equations formed by $(3.4)$ and $(3.9)$ is the main goal of this section and some remarks are required in order to proceed. For instance, the method employed here for the derivation of (3.4) and (3.9) can be seen as an extension of the method considered in [29], and the resulting functional relations indeed share some similarities with the one obtained for the partition function of the elliptic SOS 
model with domain wall boundaries. However, there are still some important structural differences that introduce some extra steps in solving $(3.4)$ and $(3.9)$.

Firstly, let us consider the similarities. The functional equations obtained here and the one of $[29]$ are all relations for a multivariate function, i.e. $F\left(z_{1}, \ldots, z_{n}\right)$, composed of a linear combination of terms involving the function $F$ with a given variable $z_{i}$ in its argument being replaced by a variable $z_{0}$. Moreover, the Eqs. $(3.4)$ and $(3.9)$ are also homogeneous in the sense that $\alpha S_{n}$ solves our system of equations if $S_{n}$ is a solution and $\alpha$ is a constant. In fact, $\alpha$ only needs to be independent of the variables $\lambda_{i}^{B}$ and $\lambda_{i}^{C}$. This property tells us in advance that Eqs. (3.4) and (3.9) will be able to determine $S_{n}$ only up to an overall multiplicative factor independent of $\lambda_{i}^{B}$ and $\lambda_{i}^{C}$ at most. Thus it will be necessary to evaluate the function $S_{n}$ for a particular value of its variables in order to have our scalar product completely fixed. Still considering the similarities, both Eqs. $(3.4)$ and $(3.9)$ are linear which raise the issue of uniqueness of the solution. Here we will be interested in a multivariate polynomial solution and this property ensures uniqueness as demonstrated in [28] under very general conditions.

Now let us consider the differences between $(3.4,3.9)$ and the functional equation obtained in [29]. The most obvious difference is that here we have obtained two equations which might suggest that one of them is redundant. However, the direct inspection of our equations for small values of $n$ and $L$ reveals that the polynomial solution which we shall be interested can not be completely fixed by only one of the equations. The situation is different when we consider both equations simultaneously, and their direct inspection shows that the system $(3.4,3.9)$ indeed determines the polynomial solution up to an overall multiplicative factor. Furthermore, in the case considered in $[29]$ we have an equation running only over one set of variables, i.e. $\left\{\lambda_{0}, \lambda_{1}, \ldots, \lambda_{n}\right\}$. Here both of our equations run over the two sets of variables $\left\{\lambda_{0}, \lambda_{1}^{B}, \ldots, \lambda_{n}^{B}\right\}$ and $\left\{\lambda_{0}, \lambda_{1}^{C}, \ldots, \lambda_{n}^{C}\right\}$. Taking into account the above discussion, the following Lemmas will pave the way for solving $(3.4,3.9)$.

Lemma 1 (Polynomial structure). In terms of variables $x_{i}^{B, C}=e^{2 \lambda_{i}^{B, C}}$, the function $S_{n}$ is of the form $S_{n}=\prod_{i=1}^{n}\left(x_{i}^{B} x_{i}^{C}\right)^{-\frac{L-1}{2}} \bar{S}_{n}\left(x_{1}^{C}, \ldots, x_{n}^{C} \mid x_{1}^{B}, \ldots, x_{n}^{B}\right)$ where $\bar{S}_{n}$ is a polynomial of order $L-1$ in each one of its variables separately.

Proof. See Appendix A.

Lemma 2 (Special zeroes). The function $S_{n}\left(\lambda_{1}^{C}, \ldots, \lambda_{n}^{C} \mid \lambda_{1}^{B}, \ldots, \lambda_{n}^{B}\right)$ vanishes for the specialisation of variables $\lambda_{1}^{B}=\mu_{1}$ and $\lambda_{2}^{B}=\mu_{1}-\gamma$. The same property also holds for the specialisation $\lambda_{1}^{C}=\mu_{1}$ and $\lambda_{2}^{C}=\mu_{1}-\gamma$.

Proof. See Appendix B.

Lemma 3 (Doubly symmetric function). The scalar product $S_{n}\left(\lambda_{1}^{C}, \ldots, \lambda_{n}^{C} \mid \lambda_{1}^{B}, \ldots, \lambda_{n}^{B}\right)$ is a symmetric function in each one of the set of variables $\left\{\lambda_{i}^{B}\right\}$ and $\left\{\lambda_{i}^{C}\right\}$ independently. More precisely,

$$
\begin{aligned}
S_{n}\left(\lambda_{1}^{C}, \ldots, \lambda_{i}^{C}, \ldots, \lambda_{j}^{C}, \ldots, \lambda_{n}^{C} \mid \lambda_{1}^{B}, \ldots,\right. & \left.\lambda_{n}^{B}\right) \\
& =S_{n}\left(\lambda_{1}^{C}, \ldots, \lambda_{j}^{C}, \ldots, \lambda_{i}^{C}, \ldots, \lambda_{n}^{C} \mid \lambda_{1}^{B}, \ldots, \lambda_{n}^{B}\right)
\end{aligned}
$$


and

$$
\begin{aligned}
S_{n}\left(\lambda_{1}^{C}, \ldots, \lambda_{n}^{C} \mid \lambda_{1}^{B}, \ldots, \lambda_{i}^{B}, \ldots, \lambda_{j}^{B}, \ldots,\right. & \left.\lambda_{n}^{B}\right) \\
& =S_{n}\left(\lambda_{1}^{C}, \ldots, \lambda_{n}^{C} \mid \lambda_{1}^{B}, \ldots, \lambda_{j}^{B}, \ldots, \lambda_{i}^{B}, \ldots, \lambda_{n}^{B}\right) .
\end{aligned}
$$

Proof. See Appendix C.

Lemma 4 (Asymptotic behaviour). In the limit $x_{i}^{B, C} \rightarrow \infty$, the function $S_{n}$ behaves as

$$
S_{n} \sim \frac{\left(q-q^{-1}\right)^{2 n}}{2^{2 n L}} q^{n(L-n)}[n !]_{q^{2}}^{2} e^{-2 n \sum_{j=1}^{L} \mu_{j}} \sum_{1 \leq a_{1}<\cdots<a_{n} \leq L} e^{2 \sum_{j=1}^{n} \mu_{a_{j}}} \prod_{i=1}^{n}\left(x_{i}^{B} x_{i}^{C}\right)^{\frac{L-1}{2}},
$$

where $[n !]_{q^{2}}$ denotes the q-factorial function defined as

$$
[n !]_{q^{2}}=1\left(1+q^{2}\right)\left(1+q^{2}+q^{4}\right) \ldots\left(1+q^{2}+\cdots+q^{2(n-1)}\right) .
$$

Proof. See Appendix D.

Remark 1. Due to the Lemma 3 we can safely employ the notation

$$
S_{n}\left(\lambda_{1}^{C}, \ldots, \lambda_{n}^{C} \mid \lambda_{1}^{B}, \ldots, \lambda_{n}^{B}\right)=S_{n}\left(X^{1, n} \mid Y^{1, n}\right)
$$

where $X^{i, j}=\left\{\lambda_{k}^{C} \mid i \leq k \leq j\right\}$ and $Y^{i, j}=\left\{\lambda_{k}^{B} \mid i \leq k \leq j\right\}$.

Except for the Lemmas 1 and 4 , the remaining ones are a direct consequence of the functional relations (3.4) and (3.9).

\subsection{Off-shell formula}

Here we shall consider the resolution of the system of equations $(\overline{3.4}, 3.9)$ for general values of variables $\lambda_{i}^{B}$ and $\lambda_{i}^{C}$. This case is refereed to as off-shell scalar product as remarked in Section 2. The methodology we shall employ for solving $(3.4,3.9)$ is similar to the one developed in [29] and in what follows we describe a sequence of steps leading to the solution.

Step 1. We firstly consider Eq. (3.4) under the specialisation of variables $\lambda_{0}=\mu_{1}-\gamma$ and $\lambda_{n}^{B}=\mu_{1}$ such that the coefficient $M_{0}$ vanishes. We shall also consider the property $S_{n}\left(X^{1, n} \mid \mu_{1}-\gamma, \ldots, \mu_{1}\right)=0$ obtained from Lemmas 2 and 3 implying the relation

$$
S_{n}\left(\bar{X}^{2, n} \mid \check{Y}^{2, n}\right)=\prod_{j=2}^{n} b\left(\lambda_{j}^{C}-\mu_{1}\right) a\left(\lambda_{j}^{B}-\mu_{1}\right) V\left(X^{2, n} \mid Y^{2, n}\right),
$$

due to Lemmas 1 and 3 . In $(4.2)$ we have also introduced the notation $\bar{Z}^{i, j}=Z^{i, j} \cup\left\{\mu_{1}-\right.$ $\gamma\}$ and $\check{Z}^{i, j}=Z^{i, j} \cup\left\{\mu_{1}\right\}$ for $Z^{i, j} \in\left\{X^{i, j}, Y^{i, j}\right\}$. In its turn the function $V$ appearing in $(4.2)$ is also of the form described in Lemma 1 under the identifications $S_{n} \mapsto V$, 
$n \mapsto n-1$ and $L \mapsto L-1$. Thus under this specialisation of variables, Eq. (3.4) yields the relation

$$
S_{n}\left(X^{1, n} \mid \bar{Y}^{1, n-1}\right)=\sum_{i=1}^{n} m_{i}^{(A)} V\left(X_{i}^{1, n} \mid Y^{1, n-1}\right),
$$

where $X_{k}^{i, j}=X^{i, j} \backslash\left\{\lambda_{k}^{C}\right\}$ and

$$
\begin{aligned}
m_{i}^{(A)} & =-\left.\prod_{j=1}^{n-1} a\left(\lambda_{j}^{B}-\mu_{1}\right) \prod_{\substack{j=1 \\
j \neq i}}^{n} b\left(\lambda_{j}^{C}-\mu_{1}\right) \frac{N_{i}^{(C)}}{N_{n}^{(B)}}\right|_{\substack{\lambda_{0}=\mu_{1}-\gamma \\
\lambda_{n}^{B}=\mu_{1}}} \prod^{L} \frac{a\left(\lambda_{i}^{C}-\mu_{j}\right)}{a-1} \prod_{j=1}^{n-1} b\left(\lambda_{j}^{B}-\mu_{1}\right) \prod_{\substack{j=1 \\
j \neq i}}^{n} b\left(\lambda_{j}^{C}-\mu_{1}\right) \frac{a\left(\lambda_{j}^{C}-\lambda_{i}^{C}\right)}{b\left(\lambda_{j}^{C}-\lambda_{i}^{C}\right)} .
\end{aligned}
$$

Step 2. Analogously to (4.2), due to Lemmas 1,2 and 3 we can write

$$
S_{n}\left(\check{X}^{2, n} \mid \bar{Y}^{2, n}\right)=\prod_{j=2}^{n} a\left(\lambda_{j}^{C}-\mu_{1}\right) b\left(\lambda_{j}^{B}-\mu_{1}\right) W\left(X^{2, n} \mid Y^{2, n}\right),
$$

where the function $W$ is also of the form described in Lemma 1 under the mappings $n \mapsto n-1$ and $L \mapsto L-1$. Then by setting $\lambda_{0}=\mu_{1}-\gamma$ and $\lambda_{n}^{C}=\mu_{1}$ in Eq. (3.4), and considering the relation $(4.5)$ in addition to the property $S_{n}\left(\mu_{1}-\gamma, \ldots, \mu_{1} \mid Y^{1, n}\right)=0$, we obtain the formula

$$
S_{n}\left(\bar{X}^{1, n-1} \mid Y^{1, n}\right)=\sum_{i=1}^{n} \bar{m}_{i}^{(A)} W\left(X^{1, n-1} \mid Y_{i}^{1, n}\right),
$$

where $Y_{k}^{i, j}=Y^{i, j} \backslash\left\{\lambda_{k}^{B}\right\}$ and

$$
\begin{aligned}
\bar{m}_{i}^{(A)} & =-\left.\prod_{j=1}^{n-1} a\left(\lambda_{j}^{C}-\mu_{1}\right) \prod_{\substack{j=1 \\
j \neq i}}^{n} b\left(\lambda_{j}^{B}-\mu_{1}\right) \frac{N_{i}^{(B)}}{N_{n}^{(C)}}\right|_{\substack{\lambda_{0}=\mu_{1}-\gamma \\
\lambda_{n}^{C}=\mu_{1}}} \prod^{L} \frac{a\left(\lambda_{i}^{B}-\mu_{j}\right)}{a-1} \prod_{j=1}^{n-1} b\left(\lambda_{j}^{C}-\mu_{1}\right) \prod_{\substack{j=1 \\
j \neq i}}^{n} b\left(\lambda_{j}^{B}-\mu_{1}\right) \frac{a\left(\lambda_{j}^{B}-\lambda_{i}^{B}\right)}{b\left(\lambda_{j}^{B}-\lambda_{i}^{B}\right)} .
\end{aligned}
$$

Step 3. The coefficient $N_{n}^{(B)}$ vanishes under the specialisation $\lambda_{n}^{B}=\mu_{1}-\gamma$. Thus, under this particular specialisation, the Eq. (3.4) will contain only terms of the form $S_{n}\left(X^{1, n} \mid \bar{Y}^{1, n-1}\right)$ allowing us to use the relation (4.3) to obtain an equation involving solely 
the function $V$. The resulting equation can be further simplified by setting $\lambda_{n}^{C}=\mu_{1}$, and in this way we are left with the relation

$$
J_{0} V\left(X^{1, n-1} \mid Y^{1, n-1}\right)+\sum_{i=1}^{n-1} K_{i}^{(B)} V\left(X^{1, n-1} \mid Y_{i}^{0, n-1}\right)+\sum_{i=1}^{n-1} K_{i}^{(C)} V\left(X_{i}^{0, n-1} \mid Y^{1, n-1}\right)=0 .
$$

In their turn the coefficients $J_{0}$ and $K_{i}^{(B, C)}$ appearing in (4.8) correspond respectively to the coefficients $M_{0}$ and $N_{i}^{(B, C)}$ given in (3.5) under the mappings $L \mapsto L-1, n \mapsto n-1$ and $\mu_{i} \mapsto \mu_{i+1}$. Thus the function $V$ obeys essentially the same equation as $S_{n-1}$ for Bethe vectors living in $\mathbb{V}_{1} \otimes \cdots \otimes \mathbb{V}_{L-1}$.

Step 4. Next we consider Eq. (3.4) with $\lambda_{n}^{C}=\mu_{1}-\gamma$. In that case the coefficient $N_{n}^{(C)}$ vanishes and we are left only with terms of the form $S_{n}\left(\bar{X}^{1, n-1} \mid Y^{1, n}\right)$. Then we use the relation (4.6) to obtain an equation only in terms of the function $W$. After setting $\lambda_{n}^{B}=\mu_{1}$ and eliminating an overall factor, the equation obtained in this way reads

$$
J_{0} W\left(X^{1, n-1} \mid Y^{1, n-1}\right)+\sum_{i=1}^{n-1} K_{i}^{(B)} W\left(X^{1, n-1} \mid Y_{i}^{0, n-1}\right)+\sum_{i=1}^{n-1} K_{i}^{(C)} W\left(X_{i}^{0, n-1} \mid Y^{1, n-1}\right)=0 .
$$

Thus the functions $V$ and $W$ obey the same equation and the uniqueness of the solution of (3.4) would imply that $V$ and $W$ can differ only by an overall constant factor.

Step 5. We consider the Eq. (3.9) with $\lambda_{0}=\mu_{1}$ and $\lambda_{n}^{B}=\mu_{1}-\gamma$. In that case the coefficient $\widetilde{M}_{0}=0$, and we can use formula $(4.5)$ in addition to the Lemma 2 to obtain the relation

$$
S_{n}\left(X^{1, n} \mid \check{Y}^{1, n-1}\right)=\sum_{i=1}^{n} \bar{m}_{i}^{(D)} W\left(X_{i}^{1, n} \mid Y^{1, n-1}\right)
$$

where

$$
\begin{aligned}
\bar{m}_{i}^{(D)} & =-\left.\prod_{j=1}^{n-1} b\left(\lambda_{j}^{B}-\mu_{1}\right) \prod_{\substack{j=1 \\
j \neq i}}^{n} a\left(\lambda_{j}^{C}-\mu_{1}\right) \frac{\widetilde{N}_{i}^{(C)}}{\widetilde{N}_{n}^{(B)}}\right|_{\substack{\lambda_{n}^{B}=\mu_{1}-\gamma \\
\lambda_{0}=\mu_{1}}} \prod^{L} \frac{b\left(\mu_{j}-\lambda_{i}^{C}\right)}{b-1} \prod_{j=1}^{n-1} a\left(\lambda_{j}^{B}-\mu_{1}\right) \prod_{\substack{j=1 \\
j \neq i}}^{n} a\left(\lambda_{j}^{C}-\mu_{1}\right) \frac{a\left(\lambda_{i}^{C}-\lambda_{j}^{C}\right)}{b\left(\lambda_{i}^{C}-\lambda_{j}^{C}\right)}
\end{aligned}
$$


Step 6. Next we set $\lambda_{0}=\mu_{1}$ and $\lambda_{n}^{C}=\mu_{1}-\gamma$ in the Eq. (3.9). This allows us to employ the relation (4.2) and the property $S_{n}\left(\mu_{1}, \ldots, \mu_{1}-\gamma \mid Y^{1, n}\right)=0$ described in Lemma 2 . In this way we obtain the expression

$$
S_{n}\left(\check{X}^{1, n-1} \mid Y^{1, n}\right)=\sum_{i=1}^{n} m_{i}^{(D)} V\left(X^{1, n-1} \mid Y_{i}^{1, n}\right)
$$

with

$$
\begin{aligned}
m_{i}^{(D)} & =-\left.\prod_{j=1}^{n-1} b\left(\lambda_{j}^{C}-\mu_{1}\right) \prod_{\substack{j=1 \\
j \neq i}}^{n} a\left(\lambda_{j}^{B}-\mu_{1}\right) \frac{\widetilde{N}_{i}^{(B)}}{\widetilde{N}_{n}^{(C)}}\right|_{\substack{\lambda_{n}^{C}=\mu_{1}-\gamma \\
\lambda_{0}=\mu_{1}}} \prod^{L} \frac{b\left(\mu_{j}-\lambda_{i}^{B}\right)}{b\left(\mu_{1}-\lambda_{i}^{B}\right)} \prod_{j=1}^{n-1} a\left(\lambda_{j}^{C}-\mu_{1}\right) \prod_{\substack{j=1 \\
j \neq i}}^{n} a\left(\lambda_{j}^{B}-\mu_{1}\right) \frac{a\left(\lambda_{i}^{B}-\lambda_{j}^{B}\right)}{b\left(\lambda_{i}^{B}-\lambda_{j}^{B}\right)} .
\end{aligned}
$$

Step 7. The Eq. (3.9) will contain only terms of the form $S_{n}\left(X^{1, n} \mid \check{Y}^{1, n-1}\right)$ for the specialisation $\lambda_{n}^{B}=\mu_{1}$ since the coefficient $\widetilde{N}_{n}^{(B)}$ vanishes. In that case we can consider the formula (4.10) and also set $\lambda_{n}^{C}=\mu_{1}-\gamma$. By doing so we are left with the relation, $\widetilde{J}_{0} W\left(X^{1, n-1} \mid Y^{1, n-1}\right)+\sum_{i=1}^{n-1} \widetilde{K}_{i}^{(B)} W\left(X^{1, n-1} \mid Y_{i}^{0, n-1}\right)+\sum_{i=1}^{n-1} \widetilde{K}_{i}^{(C)} W\left(X_{i}^{0, n-1} \mid Y^{1, n-1}\right)=0$,

whose coefficients $\widetilde{J}_{0}$ and $\widetilde{K}_{i}^{(B, C)}$ correspond respectively to the coefficients $\widetilde{M}_{0}$ and $\widetilde{N}_{i}^{(B, C)}$ given in (3.10) under the mappings $L \mapsto L-1, n \mapsto n-1$ and $\mu_{i} \mapsto \mu_{i+1}$.

Step 8. Set $\lambda_{n}^{C}=\mu_{1}$ in Eq. $(3.9)$ taking into account that the coefficient $\widetilde{N}_{n}^{(C)}$ vanishes. For this particular specialisation Eq. (3.9) contains only terms of the form $S_{n}\left(\check{X}^{1, n-1} \mid Y^{1, n}\right)$. In this way we use formula (4.12) and set $\lambda_{n}^{B}=\mu_{1}-\gamma$ to obtain the relation

$$
\widetilde{J}_{0} V\left(X^{1, n-1} \mid Y^{1, n-1}\right)+\sum_{i=1}^{n-1} \widetilde{K}_{i}^{(B)} V\left(X^{1, n-1} \mid Y_{i}^{0, n-1}\right)+\sum_{i=1}^{n-1} \widetilde{K}_{i}^{(C)} V\left(X_{i}^{0, n-1} \mid Y^{1, n-1}\right)=0 .
$$

Thus, considering Eq. (4.14) in addition to (4.15), we can see that both functions $V$ and $W$ also obeys the Eq. (3.9) under the mappings $L \mapsto L-1, n \mapsto n-1$ and $\mu_{i} \mapsto \mu_{i+1}$.

Step 9. Next we consider Eq. (3.9) with $\lambda_{0}=\mu_{1}-\gamma$ and make use of the relations (4.6) and (4.3). By doing so we obtain the expression

$$
S_{n}\left(X^{1, n} \mid Y^{1, n}\right)=\sum_{i, j=1}^{n} \frac{\Theta_{i, j}}{\mathcal{F}} V\left(X_{i}^{1, n} \mid Y_{j}^{1, n}\right)+\sum_{i, j=1}^{n} \frac{\Phi_{i, j}}{\mathcal{F}} W\left(X_{j}^{1, n} \mid Y_{i}^{1, n}\right),
$$




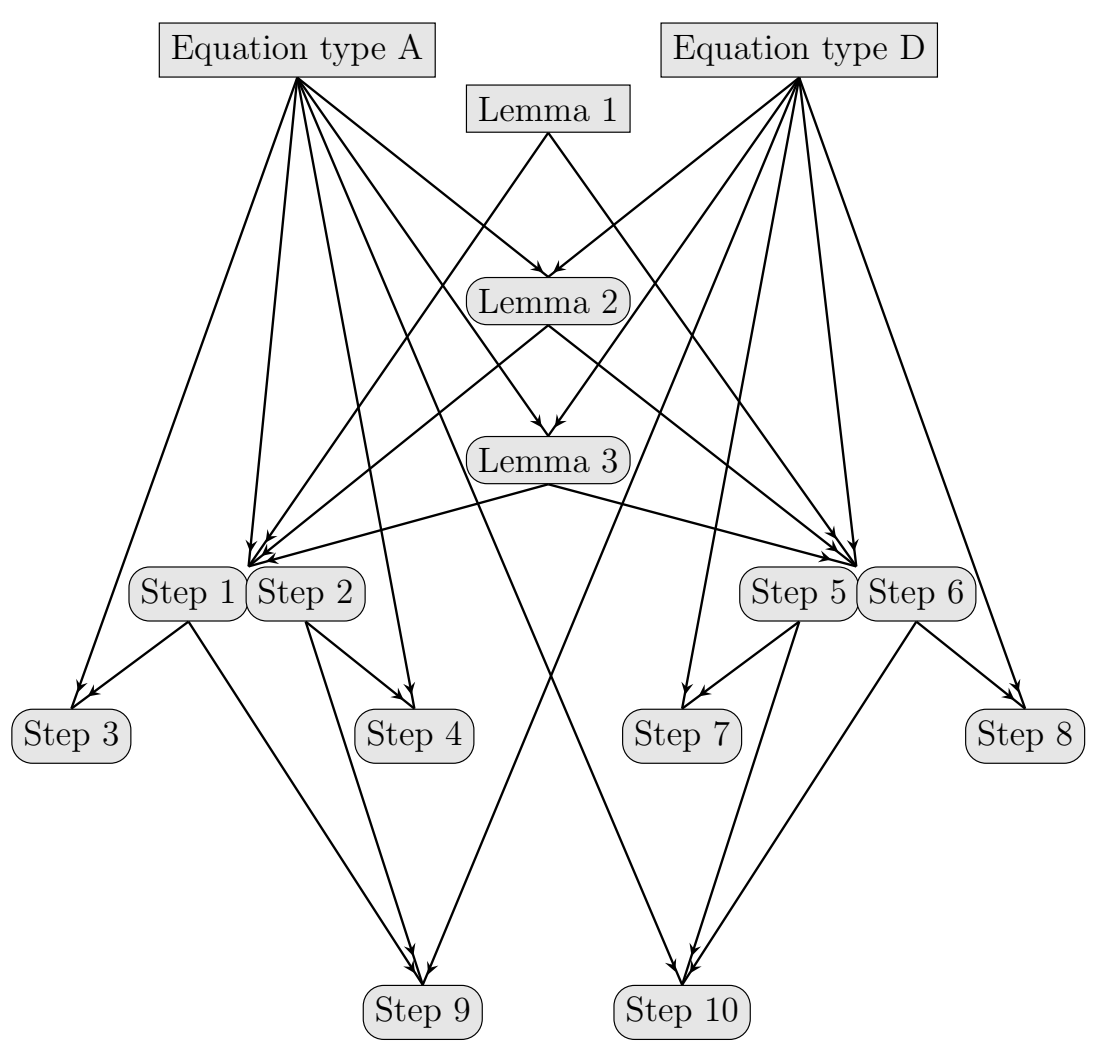

Figure 1: Interdependence among the Steps 1-10.

where

$$
\begin{aligned}
& \Theta_{i, j}= \frac{c^{2}}{b\left(\lambda_{i}^{C}-\mu_{1}\right) b\left(\mu_{1}-\lambda_{j}^{B}\right)} \prod_{k=1}^{L} \frac{a\left(\lambda_{i}^{C}-\mu_{k}\right) b\left(\mu_{k}-\lambda_{j}^{B}\right)}{a\left(\mu_{1}-\mu_{k}\right) a\left(\mu_{k}-\mu_{1}\right)} \\
& \times \prod_{\substack{k=1 \\
k \neq i}}^{n} a\left(\lambda_{k}^{C}-\mu_{1}\right) \frac{a\left(\lambda_{k}^{C}-\lambda_{i}^{C}\right)}{b\left(\lambda_{k}^{C}-\lambda_{i}^{C}\right)} \prod_{\substack{k=1 \\
k \neq j}}^{n} a\left(\lambda_{k}^{B}-\mu_{1}\right) \frac{a\left(\lambda_{j}^{B}-\lambda_{k}^{B}\right)}{b\left(\lambda_{j}^{B}-\lambda_{k}^{B}\right)} \\
& \Phi_{i, j}= \frac{c^{2}}{b\left(\lambda_{i}^{B}-\mu_{1}\right) b\left(\lambda_{j}^{C}-\mu_{1}\right)} \prod_{k=1}^{L} \frac{a\left(\lambda_{i}^{B}-\mu_{k}\right) b\left(\mu_{k}-\lambda_{j}^{C}\right)}{a\left(\mu_{1}-\mu_{k}\right) a\left(\mu_{k}-\mu_{1}\right)} \\
& \mathcal{F}= \prod_{\substack{k=1 \\
k \neq i}}^{n} a\left(\lambda_{k}^{B}-\mu_{1}\right) \frac{a\left(\lambda_{k}^{B}-\lambda_{i}^{B}\right)}{b\left(\lambda_{k}^{B}-\lambda_{i}^{B}\right)} \prod_{\substack{k=1 \\
k \neq j}}^{n} a\left(\lambda_{k}^{C}-\mu_{1}\right) \frac{a\left(\lambda_{j}^{C}-\lambda_{k}^{C}\right)}{b\left(\lambda_{j}^{C}-\lambda_{k}^{C}\right)} \\
&\left.\prod_{k}^{C}-\mu_{1}\right) \\
& \prod_{k=1}^{n} \frac{a\left(\lambda_{k}^{B}-\mu_{1}\right)}{b\left(\lambda_{k}^{B}-\mu_{1}\right)} .
\end{aligned}
$$

Step 10. For completeness we also set $\lambda_{0}=\mu_{1}$ in Eq. (3.4) and consider the expressions (4.10) and (4.12). This procedure yields the same formula (4.16). 
The interdependence among the Steps 1-10 is schematically depicted in Figure 1, and at this stage we have already gathered the main ingredients required to obtain an explicit expression for the function $S_{n}$. For instance, in the Step 9 we have obtained a formula expressing the function $S_{n}$ as a linear combination of auxiliary functions $V$ and $W$ defined respectively by $(\overline{4.2})$ and $(4.5)$. This process can be thought of as a separation of variables induced by the special zeroes described in Lemma 2. On the other hand, in the Steps 3 and 8 we have shown that the function $V$ satisfy the system of functional equations (3.4.3.9) under the mappings $L \mapsto L-1, n \mapsto n-1$ and $\mu_{i} \mapsto \mu_{i+1}$. The same holds for the function $W$ as demonstrated in the Steps 4 and 7 . Thus, since $V$ and $W$ are polynomials of the same order, the linearity of the system of equations $(3.4$ 3.9) tells us that $W=\alpha V$ where $\alpha$ is a constant. This uniqueness property employed in our argumentation has been proven in [28] under very general conditions. Moreover, since $S_{n}$ is essentially a multivariate polynomial and so is the auxiliary function $V$ due to $(4.2)$, the residues of $\left(\Theta_{i, j}+\alpha \Phi_{j, i}\right) / \mathcal{F}$ must vanish. This condition tells us that $\alpha=1$. Thus for $n \leq L$ the formula (4.16) allows us to obtain the function $S_{n}$ up to an overall multiplicative constant starting with the solution of $(3.4,3.9)$ for the case $n=1$. The solution $S_{1}\left(\lambda_{1}^{C} \mid \lambda_{1}^{B}\right)$ has been obtained in Appendix $\mathrm{E}$ and in what follows we shall demonstrate that the iteration procedure described by $(4.16)$ can be mimicked by a multiple contour integral.

Multiple contour integral. According to the above discussion the expressions $(\overline{4.16})$ and $(4.17)$ can be rewritten as

$$
S_{n}\left(X^{1, n} \mid Y^{1, n}\right)=\mathcal{K} \sum_{i, j=1}^{n} \Omega_{i, j} V\left(X_{i}^{1, n} \mid Y_{j}^{1, n}\right)
$$

where

$$
\begin{aligned}
\mathcal{K}= & \frac{\prod_{k=1}^{n} a\left(\lambda_{k}^{C}-\mu_{1}\right) a\left(\lambda_{k}^{B}-\mu_{1}\right)}{\prod_{k=2}^{L} a\left(\mu_{1}-\mu_{k}\right) a\left(\mu_{k}-\mu_{1}\right)}\left[\prod_{k=1}^{n} \frac{a\left(\lambda_{k}^{C}-\mu_{1}\right)}{b\left(\lambda_{k}^{C}-\mu_{1}\right)}-\prod_{k=1}^{n} \frac{a\left(\lambda_{k}^{B}-\mu_{1}\right)}{b\left(\lambda_{k}^{B}-\mu_{1}\right)}\right]^{-1} \\
\Omega_{i, j}= & \frac{1}{a\left(\lambda_{i}^{C}-\mu_{1}\right) b\left(\lambda_{i}^{C}-\mu_{1}\right) a\left(\lambda_{j}^{B}-\mu_{1}\right) b\left(\lambda_{j}^{B}-\mu_{1}\right)} \\
& \times\left[\prod_{k=1}^{L} a\left(\lambda_{j}^{B}-\mu_{k}\right) b\left(\mu_{k}-\lambda_{i}^{C}\right) \prod_{\substack{k=1 \\
k \neq i}}^{n} \frac{a\left(\lambda_{i}^{C}-\lambda_{k}^{C}\right)}{b\left(\lambda_{i}^{C}-\lambda_{k}^{C}\right)} \prod_{\substack{k=1 \\
k \neq j}}^{n} \frac{a\left(\lambda_{k}^{B}-\lambda_{j}^{B}\right)}{b\left(\lambda_{k}^{B}-\lambda_{j}^{B}\right)}\right. \\
& \left.-\prod_{k=1}^{L} a\left(\lambda_{i}^{C}-\mu_{k}\right) b\left(\mu_{k}-\lambda_{j}^{B}\right) \prod_{\substack{k=1 \\
k \neq i}}^{n} \frac{a\left(\lambda_{k}^{C}-\lambda_{i}^{C}\right)}{b\left(\lambda_{k}^{C}-\lambda_{i}^{C}\right)} \prod_{\substack{k=1 \\
k \neq j}}^{n} \frac{a\left(\lambda_{j}^{B}-\lambda_{k}^{B}\right)}{b\left(\lambda_{j}^{B}-\lambda_{k}^{B}\right)}\right] .
\end{aligned}
$$

Moreover, the formula (4.18) suggests that the function $S_{n}$ can be expressed as

$$
S_{n}\left(X^{1, n} \mid Y^{1, n}\right)=\oint \ldots \oint \prod_{i=1}^{n} \frac{\mathrm{d} w_{i}}{2 \mathrm{i} \pi} \frac{\mathrm{d} \bar{w}_{i}}{2 \mathrm{i} \pi} \frac{H\left(w_{1}, \ldots, w_{n} \mid \bar{w}_{1}, \ldots, \bar{w}_{n}\right)}{\prod_{i, j=1}^{n} b\left(w_{i}-\lambda_{j}^{C}\right) b\left(\bar{w}_{i}-\lambda_{j}^{B}\right)}
$$


where the integrals over the set of variables $\left\{w_{i}\right\}$ are performed around the contours enclosing solely the poles at $w_{i}=\lambda_{j}^{C}$. On the other hand, the contours associated with the integration over the set $\left\{\bar{w}_{i}\right\}$ contain only the poles at $\bar{w}_{i}=\lambda_{j}^{B}$. The function $H$ is also assumed to be independent of the variables $\lambda_{j}^{B, C}$ in such a way that the only poles contributing to the evaluation of $(4.20)$ are due to the zeroes of $\prod_{i, j=1}^{n} b\left(w_{i}-\lambda_{j}^{C}\right) b\left(\bar{w}_{i}-\right.$ $\lambda_{j}^{B}$ ). Now we can integrate the formula (4.20) over the variables $w_{1}$ and $\bar{w}_{1}$, and by doing so we generate the summations over the indexes $i$ and $j$ appearing in (4.20). This procedure allows us to look for a term by term identification, and the iteration procedure described by $(4.18)$ is realised if we are able to exhibit a function $H$ satisfying the condition

$$
\begin{aligned}
\left.H\right|_{\substack{w_{1}=\lambda_{i}^{C} \\
\bar{w}_{1}=\lambda_{j}^{B}}}= & \prod_{\substack{k=1 \\
k \neq i}}^{n} b\left(\lambda_{i}^{C}-\lambda_{k}^{C}\right) \prod_{\substack{k=1 \\
k \neq j}}^{n} b\left(\lambda_{j}^{B}-\lambda_{k}^{B}\right) \prod_{k=2}^{n} b\left(w_{k}-\lambda_{i}^{C}\right) \prod_{k=2}^{n} b\left(\bar{w}_{k}-\lambda_{j}^{B}\right) \\
& \times \mathcal{K} \Omega_{i, j} \bar{H}\left(w_{2}, \ldots, w_{n} \mid \bar{w}_{2}, \ldots, \bar{w}_{n}\right) .
\end{aligned}
$$

In its turn the function $\bar{H}$ consists of the function $H$, up to an overall multiplicative constant, under the mappings $L \mapsto L-1, n \mapsto n-1$ and $\mu_{i} \mapsto \mu_{i+1}{ }^{1}$. Also, it is important to remark here that the relation (4.21) needs to be valid for any $i, j \in[1, n]$ and that is required to hold only when integrated according to (4.20). Thus, under these requirements, we simply need to consider the relation (4.21) under the mappings $\lambda_{i}^{C} \mapsto w_{1}, \lambda_{j}^{B} \mapsto \bar{w}_{1}, \lambda_{k}^{C} \mapsto w_{k}$ for $k \neq i$ and $\lambda_{k}^{B} \mapsto \bar{w}_{k}$ for $k \neq i$ to obtain the relation

$$
\begin{aligned}
& H\left(w_{1}, \ldots, w_{n} \mid \bar{w}_{1}, \ldots, \bar{w}_{n}\right)=\frac{\bar{H}\left(w_{2}, \ldots, w_{n} \mid \bar{w}_{2}, \ldots, \bar{w}_{n}\right)}{b\left(w_{1}-\mu_{1}\right) b\left(\bar{w}_{1}-\mu_{1}\right)} \prod_{k=2}^{n} b\left(w_{1}-w_{k}\right)^{2} b\left(\bar{w}_{1}-\bar{w}_{k}\right)^{2} \\
& \times \frac{\prod_{k=2}^{n} a\left(w_{k}-\mu_{1}\right) a\left(\bar{w}_{k}-\mu_{1}\right)}{\prod_{k=2}^{L} a\left(\mu_{1}-\mu_{k}\right) a\left(\mu_{k}-\mu_{1}\right)}\left[\prod_{k=1}^{n} \frac{a\left(w_{k}-\mu_{1}\right)}{b\left(w_{k}-\mu_{1}\right)}-\prod_{k=1}^{n} \frac{a\left(\bar{w}_{k}-\mu_{1}\right)}{b\left(\bar{w}_{k}-\mu_{1}\right)}\right]^{-1} \\
& \times\left[\prod_{k=1}^{L} a\left(\bar{w}_{1}-\mu_{k}\right) b\left(\mu_{k}-w_{1}\right) \prod_{k=2}^{n} \frac{a\left(w_{1}-w_{k}\right)}{b\left(w_{1}-w_{k}\right)} \prod_{k=2}^{n} \frac{a\left(\bar{w}_{k}-\bar{w}_{1}\right)}{b\left(\bar{w}_{k}-\bar{w}_{1}\right)}\right. \\
& \left.\quad-\prod_{k=1}^{L} a\left(w_{1}-\mu_{k}\right) b\left(\mu_{k}-\bar{w}_{1}\right) \prod_{k=2}^{n} \frac{a\left(w_{k}-w_{1}\right)}{b\left(w_{k}-w_{1}\right)} \prod_{k=2}^{n} \frac{a\left(\bar{w}_{1}-\bar{w}_{k}\right)}{b\left(\bar{w}_{1}-\bar{w}_{k}\right)}\right] .
\end{aligned}
$$

Now the expression $(4.22)$ can be readily iterated once we know the function $H\left(w_{1} \mid \bar{w}_{1}\right)$. From formula (E.8) we can immediately read that ${ }^{2}$

$$
H\left(w_{1} \mid \bar{w}_{1}\right)=c^{2} \frac{\left[\prod_{k=1}^{L} a\left(w_{1}-\mu_{k}\right) b\left(\bar{w}_{1}-\mu_{k}\right)-\prod_{k=1}^{L} a\left(\bar{w}_{1}-\mu_{k}\right) b\left(w_{1}-\mu_{k}\right)\right]}{b\left(w_{1}-\mu_{1}\right) b\left(\bar{w}_{1}-\mu_{1}\right)\left[\frac{a\left(w_{1}-\mu_{1}\right)}{b\left(w_{1}-\mu_{1}\right)}-\frac{a\left(\bar{w}_{1}-\mu_{1}\right)}{b\left(\bar{w}_{1}-\mu_{1}\right)}\right]},
$$

\footnotetext{
${ }^{1}$ Strictly speaking, the relation $(4.21)$ is only required to be valid when integrated as $\oint \ldots \oint[\quad] \prod_{i=2}^{n} \mathrm{~d} w_{i} \mathrm{~d} \bar{w}_{i}$.

${ }^{2}$ Alternative contour integrals expressions are also possible for the case $n=1$. Here we have chosen $(4.23)$ in order to have a formula compatible with $(4.20)$.
} 
and the iteration of (4.22) leave us with the expression,

$$
\begin{aligned}
& H\left(w_{1}, \ldots, w_{n} \mid \bar{w}_{1}, \ldots, \bar{w}_{n}\right)= \\
& (-1)^{L n+\frac{n(n+1)}{2}} c^{2 n} \frac{\prod_{j>i}^{n} b\left(w_{i}-w_{j}\right)^{2} b\left(\bar{w}_{i}-\bar{w}_{j}\right)^{2} a\left(w_{j}-\mu_{i}\right) a\left(\bar{w}_{j}-\mu_{i}\right)}{\prod_{i=1}^{n} b\left(w_{i}-\mu_{i}\right) b\left(\bar{w}_{i}-\mu_{i}\right)} \prod_{i=1}^{n} R_{i}^{-1} \Lambda_{i},
\end{aligned}
$$

where the functions $R_{i}$ and $\Lambda_{i}$ are given by

$$
\begin{aligned}
R_{i}= & \prod_{k=i}^{n} \frac{a\left(w_{k}-\mu_{i}\right)}{b\left(w_{k}-\mu_{i}\right)}-\prod_{k=i}^{n} \frac{a\left(\bar{w}_{k}-\mu_{i}\right)}{b\left(\bar{w}_{k}-\mu_{i}\right)} \\
\Lambda_{i}= & \prod_{k=i}^{L} a\left(\bar{w}_{i}-\mu_{k}\right) b\left(\mu_{k}-w_{i}\right) \prod_{k=i+1}^{n} \frac{a\left(w_{i}-w_{k}\right)}{b\left(w_{i}-w_{k}\right)} \frac{a\left(\bar{w}_{k}-\bar{w}_{i}\right)}{b\left(\bar{w}_{k}-\bar{w}_{i}\right)} \\
& -\prod_{k=i}^{L} a\left(w_{i}-\mu_{k}\right) b\left(\mu_{k}-\bar{w}_{i}\right) \prod_{k=i+1}^{n} \frac{a\left(w_{k}-w_{i}\right)}{b\left(w_{k}-w_{i}\right)} \frac{a\left(\bar{w}_{i}-\bar{w}_{k}\right)}{b\left(\bar{w}_{i}-\bar{w}_{k}\right)} .
\end{aligned}
$$

The formula $(\overline{4.24})$ already takes into account the asymptotic behaviour described in Lemma 4.

\subsection{On-shell formula}

In what follows we shall consider the iteration of the relation $(4.18)$ when the set of variables $Y^{1, n}$ are constrained by the Bethe ansatz equations (2.13). In that case we can see from (4.19) that the term $\Omega_{i, j}$ will be the most affected and the determination of the function $H\left(w_{1}, \ldots, w_{n} \mid \bar{w}_{1}, \ldots, \bar{w}_{n}\right)$ will require some extra effort. For instance, we shall need to promote the function $H$ to $H^{(s)}\left(w_{s}, \ldots, w_{n} \mid \bar{w}_{s}, \ldots, \bar{w}_{n}\right)$ where the index $s$ is introduced in order to keep track of how many iterations we have performed. The case $s=1$ then yields the function $H$ entering in formula (4.20).

We then follow the procedure described in Section 4.1 keeping in mind that the constraint (2.13) should hold at each level of the iteration process. By doing so we find the relation,

$$
\begin{gathered}
H^{(s)}\left(w_{s}, \ldots, w_{n} \mid \bar{w}_{s}, \ldots, \bar{w}_{n}\right)=\frac{(-1)^{L} \prod_{k=1}^{L} b\left(\bar{w}_{s}-\mu_{k}\right) \prod_{k=s+1}^{n} a\left(w_{k}-\mu_{s}\right) b\left(w_{k}-w_{s}\right)}{b\left(w_{s}-\mu_{s}\right) b\left(\bar{w}_{s}-\mu_{s}\right) \prod_{k=s+1}^{L} a\left(\mu_{s}-\mu_{k}\right) a\left(\mu_{k}-\mu_{s}\right)} \\
\times \prod_{k=s+1}^{n} a\left(\bar{w}_{s}-\bar{w}_{k}\right) a\left(\bar{w}_{k}-\mu_{s}\right) b\left(\bar{w}_{k}-\bar{w}_{s}\right)\left[\prod_{k=s}^{n} \frac{a\left(w_{k}-\mu_{s}\right)}{b\left(w_{k}-\mu_{s}\right)}-\prod_{k=s}^{n} \frac{a\left(\bar{w}_{k}-\mu_{s}\right)}{b\left(\bar{w}_{k}-\mu_{s}\right)}\right]^{-1} \\
\times\left[\frac{(-1)^{n}}{\prod_{k=1}^{s-1} b\left(\bar{w}_{s}-\mu_{k}\right)} \prod_{k=s}^{L} a\left(w_{s}-\mu_{k}\right) \prod_{k=s+1}^{n} a\left(w_{k}-w_{s}\right)\right. \\
\left.\quad+\frac{\phi_{2} \phi_{1}^{-1}}{\prod_{k=1}^{s-1} a\left(\bar{w}_{s}-\mu_{k}\right)} \prod_{k=s}^{L} b\left(w_{s}-\mu_{k}\right) \prod_{k=s+1}^{n} a\left(w_{s}-w_{k}\right) \prod_{k=1}^{s-1} \frac{a\left(\bar{w}_{s}-\bar{w}_{k}\right)}{a\left(\bar{w}_{k}-\bar{w}_{s}\right)}\right]
\end{gathered}
$$




$$
\times H^{(s+1)}\left(w_{s+1}, \ldots, w_{n} \mid \bar{w}_{s+1}, \ldots, \bar{w}_{n}\right),
$$

which needs to be iterated starting from $s=1$ up to $s=n-1$. Then at the last step we shall also need the function $H^{(n)}\left(w_{n} \mid \bar{w}_{n}\right)$ which is readily obtained from (E.8) taking into account the relation $(2.13)$. Thus we have

$$
\begin{gathered}
H^{(n)}\left(w_{n} \mid \bar{w}_{n}\right)=(-1)^{n} c^{2} \frac{\prod_{k=1}^{L} b\left(\bar{w}_{n}-\mu_{k}\right)}{b\left(w_{n}-\mu_{n}\right) b\left(\bar{w}_{n}-\mu_{n}\right)}\left[\frac{a\left(w_{n}-\mu_{n}\right)}{b\left(w_{n}-\mu_{n}\right)}-\frac{a\left(\bar{w}_{n}-\mu_{n}\right)}{b\left(\bar{w}_{n}-\mu_{n}\right)}\right]^{-1} \\
\times\left[\frac{(-1)^{n}}{\prod_{k=1}^{n-1} b\left(\bar{w}_{n}-\mu_{k}\right)} \prod_{k=n}^{L} a\left(w_{n}-\mu_{k}\right)\right. \\
\left.+\frac{\phi_{2} \phi_{1}^{-1}}{\prod_{k=1}^{n-1} a\left(\bar{w}_{n}-\mu_{k}\right)} \prod_{k=n}^{L} b\left(w_{n}-\mu_{k}\right) \prod_{k=1}^{n-1} \frac{a\left(\bar{w}_{n}-\bar{w}_{k}\right)}{a\left(\bar{w}_{k}-\bar{w}_{n}\right)}\right]
\end{gathered}
$$

and the iteration process above described yields the formula

$$
\begin{aligned}
& H\left(w_{1}, \ldots, w_{n} \mid \bar{w}_{1}, \ldots, \bar{w}_{n}\right)= \\
& \quad(-1)^{(n-1)\left(L+\frac{n}{2}\right)} c^{2 n} \frac{\prod_{i=1}^{n} \prod_{j=1}^{L} b\left(\bar{w}_{i}-\mu_{j}\right) \prod_{\substack{i, j=1 \\
j>i}}^{n} a\left(\bar{w}_{i}-\bar{w}_{j}\right) a\left(\bar{w}_{j}-\mu_{i}\right) b\left(\bar{w}_{j}-\bar{w}_{i}\right)}{\prod_{i=1}^{n} b\left(\bar{w}_{i}-\mu_{i}\right)} \\
& \quad \times \frac{\prod_{\substack{i, j=1 \\
j>i}}^{n} a\left(w_{j}-\mu_{i}\right) b\left(w_{j}-w_{i}\right)}{\prod_{i=1}^{n} b\left(w_{i}-\mu_{i}\right)} \prod_{i=1}^{n} R_{i}^{-1} \Lambda_{i}^{\mathrm{ON}},
\end{aligned}
$$

where $R_{i}$ is given in $(4.25)$ and

$$
\begin{aligned}
\Lambda_{i}^{\mathrm{ON}}= & \frac{(-1)^{n}}{\prod_{k=1}^{i-1} b\left(\bar{w}_{i}-\mu_{k}\right)} \prod_{k=i}^{L} a\left(w_{i}-\mu_{k}\right) \prod_{k=i+1}^{n} a\left(w_{k}-w_{i}\right) \\
& +\frac{\phi_{2} \phi_{1}^{-1}}{\prod_{k=1}^{i-1} a\left(\bar{w}_{i}-\mu_{k}\right)} \prod_{k=i}^{L} b\left(w_{i}-\mu_{k}\right) \prod_{k=i+1}^{n} a\left(w_{i}-w_{k}\right) \prod_{k=1}^{i-1} \frac{a\left(\bar{w}_{i}-\bar{w}_{k}\right)}{a\left(\bar{w}_{k}-\bar{w}_{i}\right)} .
\end{aligned}
$$

The formulas $(4.20)$ and $(4.28)$ constitute an expression for the scalar product $S_{n}$ under the constraint (2.13). In that case we have available Slavnov's formula [35] and $(4.20,4.28)$ then corresponds to an alternative representation. Here it is also important to remark that it might still be possible to obtain simpler representations for the on-shell scalar product from the functional equations (3.4) and (3.9). For instance, notice that the quantity $\phi_{1} N_{i}^{(B)}+\phi_{2} \widetilde{N}_{i}^{(B)}$ vanishes under the condition (2.13). Thus if we multiply Eq. (3.4) by $\phi_{1}$ and add it to Eq. (3.9) multiplied by $\phi_{2}$, we are left with the relation

$$
K_{0} S_{n}\left(X^{1, n}\right)+\sum_{i=1}^{n} K_{i} S_{n}\left(X_{i}^{0, n}\right)=0
$$


with coefficients $K_{0}=\phi_{1} M_{0}+\phi_{2} \widetilde{M}_{0}$ and $K_{i}=\phi_{1} N_{i}^{(C)}+\phi_{2} \widetilde{N}_{i}^{(C)}$ for $i \in[1, n]$. In Eq. (4.30) we have omitted the dependence of $S_{n}$ with the set of variables $Y^{1, n}$ since now they are completely fixed by the relation (2.13). Moreover, we have checked for small values of $L$ and $n$ that Eq. (4.30) solely is able to determine the on-shell scalar product up to an overall multiplicative factor. Thus the direct study of $(4.30)$ might still offer the possibility of deriving alternative representations.

\section{Concluding remarks}

In this work we have derived functional relations describing the scalar product of Bethe vectors for the six-vertex model. The origin of the functional equations is a consistency condition between the Yang-Baxter algebra and the highest weight representation theory of the $\mathfrak{s l}_{2}$ algebra. More precisely, we have obtained a system formed by two equations $(3.4,3.9)$ whose resolution then yields a multiple integral representation $(4.20, \overline{4.24})$ for the aforementioned scalar product. Although a multiple integral formula for this same scalar product had been obtained previously in [36] as a specialisation of Baxter's solution of the Z-invariant six-vertex model, the structure of the formula obtained here is considerably different.

The resolution of our system of functional equations follows a simple sequence of systematic steps which are described in Section 4.1. It is worth remarking that the method employed in Section 4.1 consists of an extension of the one developed in $[28,29$ for the partition function of SOS models with domain wall boundaries.

Moreover, in Section 4.2 we have also obtained a formula for the scalar product (2.12) under the on-shell condition (2.13). In that case it is well known that the sixvertex model scalar products are given by Slavnov's determinant formula [35], and thus the formula $(4.20,4.28)$ constitutes an alternative representation. Furthermore, here we have also obtained a single functional equation describing scalar products under the on-shell condition $(4.30)$. In this way the direct study of $(4.30)$ might still offer the possibility of deriving different representations.

Recently, there has been a lot of discussion on the possibility of obtaining determinant representations for on-shell scalar products in models based on higher rank algebras [37-41]. In particular, some doubts on that possibility have been put forward in [38]. Since the method described here is based on the Yang-Baxter algebra, which is the common algebraic structure underlying integrable vertex models, it would be interesting to investigate the extension of this method for higher rank algebras together with the generalisation of the integral formulas $(4.20,4.24,4.28)$.

\section{Acknowledgements}

The author is supported by the Netherlands Organisation for Scientific Research (NWO) under the VICI grant 680-47-602. The work of W. Galleas is also part of the ERC Advanced grant research programme No. 246974, "Supersymmetry: a window to nonperturbative physics". 


\section{A Polynomial structure}

The structure of the function $S_{n}$ with a given variable $\lambda_{i}^{B}$ is completely encoded in the operator $B\left(\lambda_{i}^{B}\right)$, while its dependence with the variable $\lambda_{j}^{C}$ is described by the operator $C\left(\lambda_{j}^{C}\right)$. Thus we mainly need to characterise the dependence of the operators $B(\lambda)$ and $C(\lambda)$ with its spectral parameter in order to describe the structure of $S_{n}$ with respect to the set of variables $\left\{\lambda_{i}^{B}\right\}$ and $\left\{\lambda_{i}^{C}\right\}$. In order to proceed with this analysis it will be useful to recast formula $(2.3)$ as

$$
\mathcal{T}^{(L)}(\lambda)=\left(\begin{array}{ll}
A_{L}(\lambda) & B_{L}(\lambda) \\
C_{L}(\lambda) & D_{L}(\lambda)
\end{array}\right)
$$

where the have introduced the index $L$ to emphasise we are considering the ordered product of $L$ matrices $\mathcal{R}_{a j}$ according to (2.6). In its turn the matrix $\mathcal{R}_{a j}$ consists of a matrix in the space $\mathbb{V}_{a}$ whose entries are then matrices acting non-trivially on the $j$-space of the tensor product $\mathbb{V}_{1} \otimes \cdots \otimes \mathbb{V}_{L}$. Here $\mathbb{V}_{j} \cong \mathbb{C}^{2}$ and more precisely we have

$$
\mathcal{R}_{a j}=\left(\begin{array}{cc}
\alpha_{j} & \beta_{j} \\
\gamma_{j} & \delta_{j}
\end{array}\right)
$$

where

$$
\begin{aligned}
\alpha_{j} & =\left(\begin{array}{ll}
a & 0 \\
0 & b
\end{array}\right)_{j} & \beta_{j} & =\left(\begin{array}{ll}
0 & 0 \\
c & 0
\end{array}\right)_{j} \\
\gamma_{j} & =\left(\begin{array}{ll}
0 & c \\
0 & 0
\end{array}\right)_{j} & \delta_{j} & =\left(\begin{array}{ll}
b & 0 \\
0 & a
\end{array}\right)_{j} .
\end{aligned}
$$

Thus the construction of the operators $A, B, C$ and $D$ described in (2.6) can be implemented in a recursive manner with the help of the relation

$$
\mathcal{T}^{(L+1)}(\lambda)=\mathcal{T}^{(L)}(\lambda) \mathcal{R}_{a L+1}\left(\lambda-\mu_{L+1}\right)
$$

and initial conditions

$$
\begin{array}{ll}
A_{1}(\lambda)=\alpha_{1}\left(\lambda-\mu_{1}\right) & B_{1}(\lambda)=\beta_{1}\left(\lambda-\mu_{1}\right) \\
C_{1}(\lambda)=\gamma_{1}\left(\lambda-\mu_{1}\right) & D_{1}(\lambda)=\delta_{1}\left(\lambda-\mu_{1}\right) .
\end{array}
$$

In terms of its components the recursion relation $(\mathrm{A} .3)$ reads

$$
\begin{aligned}
& A_{L+1}(\lambda)=A_{L}(\lambda) \alpha_{L+1}\left(\lambda-\mu_{L+1}\right)+B_{L}(\lambda) \gamma_{L+1}\left(\lambda-\mu_{L+1}\right) \\
& B_{L+1}(\lambda)=A_{L}(\lambda) \beta_{L+1}\left(\lambda-\mu_{L+1}\right)+B_{L}(\lambda) \delta_{L+1}\left(\lambda-\mu_{L+1}\right) \\
& C_{L+1}(\lambda)=C_{L}(\lambda) \alpha_{L+1}\left(\lambda-\mu_{L+1}\right)+D_{L}(\lambda) \gamma_{L+1}\left(\lambda-\mu_{L+1}\right) \\
& D_{L+1}(\lambda)=C_{L}(\lambda) \beta_{L+1}\left(\lambda-\mu_{L+1}\right)+D_{L}(\lambda) \delta_{L+1}\left(\lambda-\mu_{L+1}\right)
\end{aligned}
$$

which allows us to infer the structure of the operators $A(\lambda), B(\lambda), C(\lambda)$ and $D(\lambda)$ with respect to the spectral parameter $\lambda$. For that it is important to keep in mind that the spectral parameter $\lambda$ only enters those operators through the functions $a$ and $b$ since $c$ 
depends only on the parameter $\gamma$. In terms of the variables $x=e^{2 \lambda}$ and $q=e^{\gamma}$ we have $a(x)=2^{-1} x^{-\frac{1}{2}}\left(x q-q^{-1}\right), b(x)=2^{-1} x^{-\frac{1}{2}}(x-1)$ and $c(x)=2^{-1}\left(q-q^{-1}\right)$, and in this way the entries of $\mathcal{R}_{a j}$ can be written in the form

$$
\begin{array}{ll}
\alpha_{j}(x)=2^{-1} x^{-\frac{1}{2}} P_{\alpha}(x) & \beta_{j}(x)=Q_{\beta} \\
\gamma_{j}(x)=Q_{\gamma} & \delta_{j}(x)=2^{-1} x^{-\frac{1}{2}} P_{\delta}(x) .
\end{array}
$$

The terms $P_{\alpha}(x)$ and $P_{\delta}(x)$ in $\left(\right.$ A.6) are polynomials of order 1 , while $Q_{\beta}$ and $Q_{\gamma}$ are constants. Under these considerations the iteration of $(\mathrm{A} .5)$ tells us that the operators $B(\lambda)$ and $C(\lambda)$ are of the form,

$$
B(\lambda)=x^{-\frac{(L-1)}{2}} P_{B}(x) \quad C(\lambda)=x^{-\frac{(L-1)}{2}} P_{C}(x)
$$

where $P_{B}(x)$ and $P_{C}(x)$ are both polynomials of order $L-1$. Thus the definition $(2.12)$ combined with the property (D.3) immediately tell us that the scalar product $S_{n}$ is of the form

$$
S_{n}=\prod_{i=1}^{n}\left(x_{i}^{B} x_{i}^{C}\right)^{-\frac{L-1}{2}} \bar{S}_{n}\left(x_{1}^{C}, \ldots, x_{n}^{C} \mid x_{1}^{B}, \ldots, x_{n}^{B}\right),
$$

where $x_{i}^{B, C}=e^{2 \lambda_{i}^{B, C}}$ and $\bar{S}_{n}$ is a polynomial of order $L-1$ in each one of its variables.

\section{B Special zeroes}

The method considered in Section 4.1 for solving the system of Eqs. $(3.4,3.9)$ relies on the identification of certain zeroes of $S_{n}$ as function of the set of variables $\left\{\lambda_{i}^{B}\right\}$ and $\left\{\lambda_{i}^{C}\right\}$. This is due to the fact that we are interested in a polynomial solution, and as such it can be characterised by its zeroes. In what follows we shall proceed with the identification of those zeroes for particular values of $n$ for illustrative purposes, and subsequently consider the general case.

\section{B.1 Case $n=2$}

The coefficients $N_{1}^{(C)}$ and $N_{2}^{(C)}$ vanish for the specialisation $\lambda_{1}^{C}=\mu_{1}$ and $\lambda_{2}^{C}=\mu_{1}-\gamma$. Thus for this particular choice of variables $\lambda_{1,2}^{C}$, Eq. (3.4) simplifies to

$$
M_{0} S_{2}\left(\mu_{1}, \mu_{1}-\gamma \mid \lambda_{1}^{B}, \lambda_{2}^{B}\right)+N_{1}^{B} S_{2}\left(\mu_{1}, \mu_{1}-\gamma \mid \lambda_{0}, \lambda_{2}^{B}\right)+N_{2}^{B} S_{2}\left(\mu_{1}, \mu_{1}-\gamma \mid \lambda_{0}, \lambda_{1}^{B}\right)=0 .
$$

Next we set $\lambda_{0}=\mu_{1}-\gamma$ noticing that Eq. (B.1) does not simplify significantly. Nevertheless, the function $M_{0}$ then consists of a single term. In this way we obtain the following relation,

$$
\begin{aligned}
S_{2}\left(\mu_{1}, \mu_{1}-\gamma \mid \lambda_{1}^{B}, \lambda_{2}^{B}\right)= & -\frac{N_{1}^{(B)}}{M_{0}} S_{2}\left(\mu_{1}, \mu_{1}-\gamma \mid \mu_{1}-\gamma, \lambda_{2}^{B}\right) \\
& -\frac{N_{2}^{(B)}}{M_{0}} S_{2}\left(\mu_{1}, \mu_{1}-\gamma \mid \mu_{1}-\gamma, \lambda_{1}^{B}\right)
\end{aligned}
$$


Eq. (3.9) also simplifies similarly under the specialisation $\lambda_{1}^{C}=\mu_{1}$ and $\lambda_{2}^{C}=\mu_{1}-\gamma$. In that case it reads,

$$
\widetilde{M}_{0} S_{2}\left(\mu_{1}, \mu_{1}-\gamma \mid \lambda_{1}^{B}, \lambda_{2}^{B}\right)+\widetilde{N}_{1}^{B} S_{2}\left(\mu_{1}, \mu_{1}-\gamma \mid \lambda_{0}, \lambda_{2}^{B}\right)+\widetilde{N}_{2}^{B} S_{2}\left(\mu_{1}, \mu_{1}-\gamma \mid \lambda_{0}, \lambda_{1}^{B}\right)=0,
$$

and we can substitute $(\overline{B .2})$ into $(\overline{B .3})$ to obtain

$$
\begin{aligned}
& Q_{0} S_{2}\left(\mu_{1}, \mu_{1}-\gamma \mid \mu_{1}-\gamma, \lambda_{0}\right) \\
& +Q_{1} S_{2}\left(\mu_{1}, \mu_{1}-\gamma \mid \mu_{1}-\gamma, \lambda_{1}^{B}\right)+Q_{2} S_{2}\left(\mu_{1}, \mu_{1}-\gamma \mid \mu_{1}-\gamma, \lambda_{2}^{B}\right)=0 .
\end{aligned}
$$

In terms of variables $x_{i}^{B}=e^{2 \lambda_{i}^{B}}$, the functions $Q_{i}$ are rational functions whose explicit forms are not enlightening. Nevertheless, they possess very non-trivial zeroes and by setting $\lambda_{i}^{B}=\left\{r_{i} \mid Q_{i}\left(r_{i}\right)=0\right\}$ for $i=1,2$ we are left with the relation

$$
\left.Q_{0}\right|_{\substack{\lambda_{1}^{B}=r_{1} \\ \lambda_{2}^{B}=r_{2}}} S_{2}\left(\mu_{1}, \mu_{1}-\gamma \mid \mu_{1}-\gamma, \lambda_{0}\right)=0 .
$$

Thus we can conclude that $S_{2}\left(\mu_{1}, \mu_{1}-\gamma \mid \mu_{1}-\gamma, \lambda_{0}\right)$ vanishes and by taking this result back to (B.2) we obtain $S_{2}\left(\mu_{1}, \mu_{1}-\gamma \mid \lambda_{1}^{B}, \lambda_{2}^{B}\right)=0$. The same procedure can be employed considering initially the variables $\lambda_{i}^{B}$ instead of $\lambda_{i}^{C}$, and by doing so we end up with the property $S_{2}\left(\lambda_{1}^{C}, \lambda_{2}^{C} \mid \mu_{1}, \mu_{1}-\gamma\right)=0$.

\section{B.2 Case $n=3$}

We set $\lambda_{1}^{C}=\mu_{1}$ and $\lambda_{2}^{C}=\mu_{1}-\gamma$ in Eq. (3.4) such that $N_{1}^{(C)}=N_{2}^{(C)}=0$. In that case (3.4) simplifies to

$$
\begin{aligned}
& M_{0} S_{3}\left(\mu_{1}, \mu_{1}-\gamma, \lambda_{3}^{C} \mid \lambda_{1}^{B}, \lambda_{2}^{B}, \lambda_{3}^{B}\right)+N_{3}^{(C)} S_{3}\left(\lambda_{0}, \mu_{1}, \mu_{1}-\gamma \mid \lambda_{1}^{B}, \lambda_{2}^{B}, \lambda_{3}^{B}\right) \\
& +N_{1}^{(B)} S_{3}\left(\mu_{1}, \mu_{1}-\gamma, \lambda_{3}^{C} \mid \lambda_{0}, \lambda_{2}^{B}, \lambda_{3}^{B}\right)+N_{2}^{(B)} S_{3}\left(\mu_{1}, \mu_{1}-\gamma, \lambda_{3}^{C} \mid \lambda_{0}, \lambda_{1}^{B}, \lambda_{3}^{B}\right) \\
& +N_{3}^{(B)} S_{3}\left(\mu_{1}, \mu_{1}-\gamma, \lambda_{3}^{C} \mid \lambda_{0}, \lambda_{1}^{B}, \lambda_{2}^{B}\right)=0 .
\end{aligned}
$$

Next we set $\lambda_{1}^{B}=\mu_{1}-\gamma$ and $\lambda_{2}^{B}=\mu_{1}$ in $($ B.6) and obtain the relation

$$
\begin{aligned}
& M_{0} S_{3}\left(\mu_{1}, \mu_{1}-\gamma, \lambda_{3}^{C} \mid \mu_{1}-\gamma, \mu_{1}, \lambda_{3}^{B}\right)+N_{3}^{(C)} S_{3}\left(\lambda_{0}, \mu_{1}, \mu_{1}-\gamma \mid \mu_{1}-\gamma, \mu_{1}, \lambda_{3}^{B}\right) \\
& +N_{3}^{(B)} S_{3}\left(\mu_{1}, \mu_{1}-\gamma, \lambda_{3}^{C} \mid \lambda_{0}, \mu_{1}-\gamma, \mu_{1}\right)=0
\end{aligned}
$$

In addition to that we set $\lambda_{0}=\mu_{1}-\gamma$ in (B.7) which then yields the following relation,

$$
\begin{aligned}
& M_{0} S_{3}\left(\mu_{1}, \mu_{1}-\gamma, \lambda_{3}^{C} \mid \mu_{1}-\gamma, \mu_{1}, \lambda_{3}^{B}\right)+N_{3}^{(C)} S_{3}\left(\mu_{1}-\gamma, \mu_{1}, \mu_{1}-\gamma \mid \mu_{1}-\gamma, \mu_{1}, \lambda_{3}^{B}\right) \\
& +N_{3}^{(B)} S_{3}\left(\mu_{1}, \mu_{1}-\gamma, \lambda_{3}^{C} \mid \mu_{1}-\gamma, \mu_{1}-\gamma, \mu_{1}\right)=0 .
\end{aligned}
$$

Furthermore, the relation $(\mathrm{B} .8)$ simplifies to

$$
S_{3}\left(\mu_{1}-\gamma, \mu_{1}, \mu_{1}-\gamma \mid \mu_{1}-\gamma, \mu_{1}, \lambda\right)=S_{3}\left(\mu_{1}, \mu_{1}-\gamma, \lambda \mid \mu_{1}-\gamma, \mu_{1}-\gamma, \mu_{1}\right),
$$


for $\lambda_{3}^{B}=\lambda_{3}^{C}=\lambda$ since the coefficient $M_{0}$ is proportional to $b\left(\lambda_{3}^{C}-\lambda_{3}^{B}\right)$. Then we can see that Eq. (B.8) combined with the property (B.9) yields the relation

$$
\begin{aligned}
S_{3}\left(\mu_{1}, \mu_{1}-\gamma, \lambda_{3}^{C} \mid \mu_{1}-\gamma, \mu_{1}, \lambda_{3}^{B}\right)= & -\frac{N_{3}^{(C)}}{M_{0}} S_{3}\left(\mu_{1}-\gamma, \mu_{1}, \mu_{1}-\gamma \mid \mu_{1}-\gamma, \mu_{1}, \lambda_{3}^{B}\right) \\
& -\frac{N_{3}^{(B)}}{M_{0}} S_{3}\left(\mu_{1}-\gamma, \mu_{1}, \mu_{1}-\gamma \mid \mu_{1}-\gamma, \mu_{1}, \lambda_{3}^{C}\right),
\end{aligned}
$$

which can be substituted back into (B.7) considering the symmetry property (3). We are then left with an equation of the form

$$
\begin{aligned}
& Q_{0} S_{3}\left(\mu_{1}-\gamma, \mu_{1}, \mu_{1}-\gamma \mid \mu_{1}-\gamma, \mu_{1}, \lambda_{0}\right)+Q_{3}^{B} S_{3}\left(\mu_{1}-\gamma, \mu_{1}, \mu_{1}-\gamma \mid \mu_{1}-\gamma, \mu_{1}, \lambda_{3}^{B}\right) \\
& +Q_{3}^{C} S_{3}\left(\mu_{1}-\gamma, \mu_{1}, \mu_{1}-\gamma \mid \mu_{1}-\gamma, \mu_{1}, \lambda_{3}^{C}\right)=0,
\end{aligned}
$$

where $Q_{0}$ and $Q_{3}^{B, C}$ are rational functions in the variables $x_{i}^{B, C}$. Then we can set $\lambda_{3}^{B, C}=$ $\left\{r^{B, C} \mid Q_{3}^{B, C}\left(r^{B, C}\right)=0\right\}$ in (B.11) and conclude that $S_{3}\left(\mu_{1}-\gamma, \mu_{1}, \mu_{1}-\gamma \mid \mu_{1}-\gamma, \mu_{1}, \lambda_{0}\right)=$ 0 . Considering (B.10), the latter also implies in

$$
S_{3}\left(\mu_{1}, \mu_{1}-\gamma, \lambda_{3}^{C} \mid \mu_{1}-\gamma, \mu_{1}, \lambda_{3}^{B}\right)=0 .
$$

Next we set $\lambda_{0}=\mu_{1}$ and $\lambda_{1}^{B}=\mu_{1}-\gamma$ in Eq. (3.4) considering the property (B.12). By doing so we obtain the following relation,

$$
M_{0} S_{3}\left(\mu_{1}, \mu_{1}-\gamma, \lambda_{3}^{C} \mid \mu_{1}-\gamma, \lambda_{2}^{B}, \lambda_{3}^{B}\right)+N_{3}^{(C)} S_{3}\left(\mu_{1}, \mu_{1}, \mu_{1}-\gamma \mid \mu_{1}-\gamma, \lambda_{2}^{B}, \lambda_{3}^{B}\right)=0 .
$$

The coefficient $M_{0}$ in (B.13) vanishes for $\lambda_{3}^{C}=\mu_{1}-\gamma$ while $N_{3}^{(C)}$ is finite. Thus for this particular specialisation of $\lambda_{3}^{C}$ we obtain $S_{3}\left(\mu_{1}, \mu_{1}, \mu_{1}-\gamma \mid \mu_{1}-\gamma, \lambda_{2}^{B}, \lambda_{3}^{B}\right)=0$, and consequently we can conclude that

$$
S_{3}\left(\mu_{1}, \mu_{1}-\gamma, \lambda_{3}^{C} \mid \mu_{1}-\gamma, \lambda_{2}^{B}, \lambda_{3}^{B}\right)=0 .
$$

So far we have only considered Eq. (3.4) and the next step is to set $\lambda_{1}^{C}=\mu_{1}$, $\lambda_{2}^{C}=\mu_{1}-\gamma$ and $\lambda_{0}=\mu_{1}-\gamma$ in Eq. (3.9). We also take into account the property (B.14) and by doing so we obtain the relation

$$
\widetilde{M}_{0} S_{3}\left(\mu_{1}, \mu_{1}-\gamma, \lambda_{3}^{C} \mid \lambda_{1}^{B}, \lambda_{2}^{B}, \lambda_{3}^{B}\right)+\widetilde{N}_{3}^{(C)} S_{3}\left(\mu_{1}-\gamma, \mu_{1}, \mu_{1}-\gamma \mid \lambda_{1}^{B}, \lambda_{2}^{B}, \lambda_{3}^{B}\right)=0 .
$$

The function $\widetilde{M}_{0}$ possesses non-trivial zeroes and we can set $\lambda_{3}^{C}$ in (B.15) such that $\widetilde{M}_{0}$ vanishes in order to conclude that $S_{3}\left(\mu_{1}-\gamma, \mu_{1}, \mu_{1}-\gamma \mid \lambda_{1}^{B}, \lambda_{2}^{B}, \lambda_{3}^{B}\right)=0$. Then from (B.15) the latter implies in

$$
S_{3}\left(\mu_{1}, \mu_{1}-\gamma, \lambda_{3}^{C} \mid \lambda_{1}^{B}, \lambda_{2}^{B}, \lambda_{3}^{B}\right)=0 .
$$

The procedure above described can also be performed with the specialisations of the variables $\lambda_{i}^{B}$ and $\lambda_{i}^{C}$ exchanged allowing us to conclude that

$$
S_{3}\left(\lambda_{1}^{C}, \lambda_{2}^{C}, \lambda_{3}^{C} \mid \mu_{1}, \mu_{1}-\gamma, \lambda_{3}^{B}\right)=0 .
$$




\section{B.3 General case}

We consider Eq. (3.4) under the specialisations $\lambda_{1}^{C}=\lambda_{2}^{B}=\mu_{1}, \lambda_{2}^{C}=\lambda_{1}^{B}=\mu_{1}-\gamma$ and $\lambda_{j}^{B, C}=\lambda_{j+1}^{B, C}+\gamma$ for $j \in[3, n-1]$ and collect the results at each step. At the final step the only non-vanishing coefficients are $M_{0}$ and $N_{n}^{(B, C)}$, and we then set $\lambda_{0}=\mu_{1}-\gamma$. This procedure then yields the formula,

$$
S_{n}(X \mid Y)=-\frac{N_{n}^{(C)}}{M_{0}} S_{n}\left(X^{*} \mid Y\right)-\frac{N_{n}^{(B)}}{M_{0}} S_{n}\left(X \mid Y^{*}\right)
$$

where

$$
\begin{aligned}
X & =\left\{\mu_{1}, \mu_{1}-\gamma, \lambda_{n}^{C}+(n-3) \gamma, \ldots, \lambda_{n}^{C}+(n-j) \gamma, \ldots, \lambda_{n}^{C}\right\} \\
Y & =\left\{\mu_{1}-\gamma, \mu_{1}, \lambda_{n}^{B}+(n-3) \gamma, \ldots, \lambda_{n}^{B}+(n-j) \gamma, \ldots, \lambda_{n}^{B}\right\} \\
X^{*} & =\left\{\mu_{1}-\gamma, \mu_{1}, \mu_{1}-\gamma, \lambda_{n}^{C}+(n-3) \gamma, \ldots, \lambda_{n}^{C}+(n-j) \gamma, \ldots, \lambda_{n}^{C}+\gamma\right\} \\
Y^{*} & =\left\{\mu_{1}-\gamma, \mu_{1}-\gamma, \mu_{1}, \lambda_{n}^{B}+(n-3) \gamma, \ldots, \lambda_{n}^{B}+(n-j) \gamma, \ldots, \lambda_{n}^{B}+\gamma\right\} .
\end{aligned}
$$

The relation (B.18) can now be substituted back into the previous steps leading to it, and an analysis similar to the one employed for the cases $n=2,3$ allows us to conclude that

$$
S_{n}\left(\mu_{1}, \mu_{1}-\gamma, \lambda_{3}^{C}, \ldots, \lambda_{n}^{C} \mid \mu_{1}-\gamma, \lambda_{2}^{B}, \ldots, \lambda_{n}^{B}\right)=0 .
$$

Next we set $\lambda_{1}^{C}=\mu_{1}, \lambda_{2}^{C}=\mu_{1}-\gamma$ and $\lambda_{0}=\mu_{1}-\gamma$ in Eq. (3.9) taking into account the property (B.20). By doing so we obtain the more general condition

$$
S_{n}\left(\mu_{1}, \mu_{1}-\gamma, \lambda_{3}^{C}, \ldots, \lambda_{n}^{C} \mid \lambda_{1}^{B}, \ldots, \lambda_{n}^{B}\right)=0 .
$$

The procedure above described can also be performed with the specialisations of the variables $\lambda_{i}^{B}$ and $\lambda_{i}^{C}$ exchanged. In that case we then obtain the vanishing condition

$$
S_{n}\left(\lambda_{1}^{C}, \ldots, \lambda_{n}^{C} \mid \mu_{1}, \mu_{1}-\gamma, \lambda_{3}^{B}, \ldots, \lambda_{n}^{B}\right)=0 .
$$

\section{C $\quad S_{n}$ as a doubly symmetric function}

The function $S_{n}\left(\lambda_{1}^{C}, \ldots, \lambda_{n}^{C} \mid \lambda_{1}^{B}, \ldots, \lambda_{n}^{B}\right)$ defined by $(2.12)$ is expected to be invariant under the exchange of variables $\lambda_{i}^{B} \leftrightarrow \lambda_{j}^{B}$ and $\lambda_{i}^{C} \leftrightarrow \lambda_{j}^{C}$ separately. This property is due to the definition (2.12) and the commutation relations

$$
\left[B\left(\lambda_{1}\right), B\left(\lambda_{2}\right)\right]=\left[C\left(\lambda_{1}\right), C\left(\lambda_{2}\right)\right]=0
$$

described in (3.2) and (3.3). Nevertheless, once we assume $S_{n}$ is determined by the Eqs. (3.4) and (3.9), it would be desirable this symmetry to be an inherent property of their solutions. This is indeed the case and it can be demonstrated as follows. 
We firstly notice that the coefficients $M_{0}$ and $N_{i}^{(B)}$ defined in (3.5) are the only coefficients exhibiting poles when $\lambda_{0} \rightarrow \lambda_{i}^{B}$. Moreover, those coefficients also satisfy the property

$$
\begin{aligned}
\lim _{\lambda_{0} \rightarrow \lambda_{i}^{B}} M_{0} b\left(\lambda_{0}-\lambda_{i}^{B}\right) & =-\lim _{\lambda_{0} \rightarrow \lambda_{i}^{B}} N_{i}^{B} b\left(\lambda_{0}-\lambda_{i}^{B}\right) \\
& =c \prod_{j=1}^{L} a\left(\lambda_{i}^{B}-\mu_{j}\right) \prod_{\substack{j=1 \\
j \neq i}}^{n} \frac{a\left(\lambda_{j}^{B}-\lambda_{i}^{B}\right)}{b\left(\lambda_{j}^{B}-\lambda_{i}^{B}\right)} .
\end{aligned}
$$

Next we integrate Eq. (3.4) over the variable $\lambda_{0}$ and around the contour $\mathcal{C}_{i}^{B}$ enclosing solely the variable $\lambda_{i}^{B}$. Thus considering the property (C.2) we obtain the identity

$$
\begin{aligned}
c \prod_{j=1}^{L} a\left(\lambda_{i}^{B}-\mu_{j}\right) \prod_{\substack{j=1 \\
j \neq i}}^{n} \frac{a\left(\lambda_{j}^{B}-\lambda_{i}^{B}\right)}{b\left(\lambda_{j}^{B}-\lambda_{i}^{B}\right)}\left[S_{n}\left(\lambda_{1}^{C}, \ldots, \lambda_{n}^{C} \mid \lambda_{1}^{B}, \ldots, \lambda_{n}^{B}\right)\right. \\
\left.\quad-\quad S_{n}\left(\lambda_{1}^{C}, \ldots, \lambda_{n}^{C} \mid \lambda_{i}^{B}, \lambda_{1}^{B}, \ldots, \lambda_{i-1}^{B}, \lambda_{i+1}^{B}, \ldots, \lambda_{n}^{B}\right)\right]=0 .
\end{aligned}
$$

The formula (C.3) allows us to conclude that

$$
S_{n}\left(\lambda_{1}^{C}, \ldots, \lambda_{n}^{C} \mid \lambda_{i}^{B}, \lambda_{1}^{B}, \ldots, \lambda_{i-1}^{B}, \lambda_{i+1}^{B}, \ldots, \lambda_{n}^{B}\right)=S_{n}\left(\lambda_{1}^{C}, \ldots, \lambda_{n}^{C} \mid \lambda_{1}^{B}, \ldots, \lambda_{n}^{B}\right)
$$

for $i \in[1, L]$, which implies the symmetry relation

$$
S_{n}\left(\lambda_{1}^{C}, \ldots, \lambda_{n}^{C} \mid \ldots, \lambda_{i}^{B}, \ldots, \lambda_{j}^{B}, \ldots\right)=S_{n}\left(\lambda_{1}^{C}, \ldots, \lambda_{n}^{C} \mid \ldots, \lambda_{j}^{B}, \ldots, \lambda_{i}^{B}, \ldots\right) .
$$

Now in order to demonstrate an equivalent symmetry relation with respect to the set of variables $\left\{\lambda_{i}^{C}\right\}$, we integrate Eq. (3.4) over the variable $\lambda_{0}$ and around the contour $\mathcal{C}_{i}^{C}$ containing solely the variable $\lambda_{i}^{C}$. We also take into account that the coefficients $M_{0}$ and $N_{i}^{(C)}$ are the only ones possessing poles when $\lambda_{0} \rightarrow \lambda_{i}^{C}$. In this way we obtain the identity

$$
\begin{aligned}
c \prod_{j=1}^{L} a\left(\lambda_{i}^{C}-\mu_{j}\right) \prod_{\substack{j=1 \\
j \neq i}}^{n} \frac{a\left(\lambda_{j}^{C}-\lambda_{i}^{C}\right)}{b\left(\lambda_{j}^{C}-\lambda_{i}^{C}\right)}\left[S_{n}\left(\lambda_{1}^{C}, \ldots, \lambda_{n}^{C} \mid \lambda_{1}^{B}, \ldots, \lambda_{n}^{B}\right)\right. \\
\left.\quad-\quad S_{n}\left(\lambda_{i}^{C}, \lambda_{1}^{C}, \ldots, \lambda_{i-1}^{C}, \lambda_{i+1}^{C}, \ldots, \lambda_{n}^{C} \mid \lambda_{1}^{B}, \ldots, \lambda_{n}^{B}\right)\right]=0,
\end{aligned}
$$

with the help of the property

$$
\begin{aligned}
\lim _{\lambda_{0} \rightarrow \lambda_{i}^{C}} M_{0} b\left(\lambda_{0}-\lambda_{i}^{C}\right) & =-\lim _{\lambda_{0} \rightarrow \lambda_{i}^{C}} N_{i}^{(C)} b\left(\lambda_{0}-\lambda_{i}^{C}\right) \\
& =-c \prod_{j=1}^{L} a\left(\lambda_{i}^{C}-\mu_{j}\right) \prod_{\substack{j=1 \\
j \neq i}}^{n} \frac{a\left(\lambda_{j}^{C}-\lambda_{i}^{C}\right)}{b\left(\lambda_{j}^{C}-\lambda_{i}^{C}\right)} .
\end{aligned}
$$


The property $S_{n}\left(\lambda_{i}^{C}, \lambda_{1}^{C}, \ldots, \lambda_{i-1}^{C}, \lambda_{i+1}^{C}, \ldots, \lambda_{n}^{C} \mid \lambda_{1}^{B}, \ldots, \lambda_{n}^{B}\right)=S_{n}\left(\lambda_{1}^{C}, \ldots, \lambda_{n}^{C} \mid \lambda_{1}^{B}, \ldots, \lambda_{n}^{B}\right)$ is then valid for $i \in[1, L]$ which allows us to conclude that

$$
S_{n}\left(\ldots, \lambda_{i}^{C}, \ldots, \lambda_{j}^{C}, \ldots \mid \lambda_{1}^{B}, \ldots, \lambda_{n}^{B}\right)=S_{n}\left(\ldots, \lambda_{j}^{C}, \ldots, \lambda_{i}^{C}, \ldots \mid \lambda_{1}^{B}, \ldots, \lambda_{n}^{B}\right) .
$$

It is important to remark here that the symmetry relations $(\overline{C .5})$ and $(\overline{C .8})$ have been obtained solely from the examination of the Eq. (3.4). Alternatively, we could also have derived the same symmetry relations from a similar analysis of the Eq. (3.9).

\section{Asymptotic behaviour}

Considering the variables $x=e^{2 \lambda}, q=e^{\gamma}$ and the conventions described in (A.2) and (A.2), in the limit $x \rightarrow \infty$ we find

$$
\begin{array}{ll}
\alpha \sim \frac{x^{\frac{1}{2}} q^{\frac{1}{2}}}{2} K & \beta \sim \frac{q-q^{-1}}{2} X^{-} \\
\gamma \sim \frac{q-q^{-1}}{2} X^{+} & \delta \sim \frac{x^{\frac{1}{2}} q^{\frac{1}{2}}}{2} K^{-1},
\end{array}
$$

where the operators $K$ and $X^{ \pm}$are explicitly given by

$$
K=\left(\begin{array}{cc}
q^{\frac{1}{2}} & 0 \\
0 & q^{-\frac{1}{2}}
\end{array}\right) \quad X^{ \pm}=\frac{1}{2}\left(\begin{array}{cc}
0 & 1 \pm 1 \\
1 \mp 1 & 0
\end{array}\right) .
$$

In this particular limit the relations $(\mathrm{A.5})$ can be easily iterated and we obtain

$$
\begin{aligned}
& B(\lambda) \sim \frac{\left(q-q^{-1}\right)}{2} q^{\frac{L-1}{2}} x^{\frac{L-1}{2}} e^{-\sum_{j=1}^{L} \mu_{j}} \sum_{k=1}^{L} e^{\mu_{k}} P_{k}^{-} \\
& C(\lambda) \sim \frac{\left(q-q^{-1}\right)}{2} q^{\frac{L-1}{2}} x^{\frac{L-1}{2}} e^{-\sum_{j=1}^{L} \mu_{j}} \sum_{k=1}^{L} e^{\mu_{k}} P_{k}^{+}
\end{aligned}
$$

with operators $P_{j}^{ \pm}$being defined as

$$
P_{j}^{ \pm}=\bigotimes_{k=1}^{j-1} K^{\mp 1} \otimes X^{ \pm} \otimes \bigotimes_{k=j+1}^{L} K^{ \pm 1} .
$$

In their turn the operators $K^{ \pm 1}$ and $X^{ \pm}$satisfy the $q$-deformed $\mathfrak{s u}(2)$ algebra,

$$
\begin{aligned}
K X^{ \pm} K^{-1} & =q^{ \pm 1} X^{ \pm} \\
{\left[X^{+}, X^{-}\right] } & =\frac{K^{2}-K^{-2}}{q-q^{-1}},
\end{aligned}
$$

which allows us to demonstrate the relations

$$
\begin{aligned}
P_{i}^{a} P_{j}^{b} & =q^{(a, b)} P_{j}^{b} P_{i}^{a} \quad \quad(i<j) \\
\left(P_{i}^{a}\right)^{2} & =0
\end{aligned}
$$


with symbols $(a, b)$ being defined as

$$
(a, b)= \begin{cases}2 & a= \pm, b= \pm \\ -2 & a= \pm, b=\mp\end{cases}
$$

Now with the help of the relations (D.6) and considering $x_{i}=e^{2 \lambda_{i}}$, in the limit $x_{i} \rightarrow \infty$ we obtain the expression

$$
\begin{array}{r}
\prod_{i=1}^{n} B\left(x_{i}\right) \sim \frac{\left(q-q^{-1}\right)^{n}}{2^{n L}} q^{n \frac{(L-1)}{2}-n(n-1)}[n !]_{q^{2}} e^{-n \sum_{j=1}^{L} \mu_{j}} \prod_{i=1}^{n} x_{i}^{\frac{L-1}{2}} \\
\times \sum_{1 \leq a_{1}<\cdots<a_{n} \leq L} e^{\sum_{j=1}^{n} \mu_{a_{j}}} \prod_{1 \leq j \leq n}^{\longrightarrow} P_{a_{j}}^{-}
\end{array}
$$

and similarly

$$
\begin{array}{r}
\prod_{i=1}^{n} C\left(x_{i}\right) \sim \frac{\left(q-q^{-1}\right)^{n}}{2^{n L}} q^{n \frac{(L-1)}{2}-n(n-1)}[n !]_{q^{2}} e^{-n \sum_{j=1}^{L} \mu_{j}} \prod_{i=1}^{n} x_{i}^{\frac{L-1}{2}} \\
\times \sum_{1 \leq a_{1}<\cdots<a_{n} \leq L} e^{\sum_{j=1}^{n} \mu_{a_{j}}} \prod_{1 \leq j \leq n}^{\longrightarrow} P_{a_{j}}^{+} .
\end{array}
$$

The term $[n !]_{q^{2}}$ appearing in $(\overline{\mathrm{D} .8})$ and $(\overline{\mathrm{D} .9})$ corresponds to the $q$-factorial function and it is defined as $[n !]_{q^{2}}=1\left(1+q^{2}\right)\left(1+q^{2}+q^{4}\right) \ldots\left(1+q^{2}+\cdots+q^{2(n-1)}\right)$.

The relations (D.8) and (D.9) can now be combined according to (2.12) to obtain the following formula,

$$
\begin{aligned}
S_{n}\left(x_{1}^{C}, \ldots, x_{n}^{C} \mid x_{1}^{B}, \ldots, x_{n}^{B}\right) \sim & \frac{\left(q-q^{-1}\right)^{2 n}}{2^{2 n L}} q^{n(L-1)-2 n(n-1)}[n !]_{q^{2}}^{2} e^{-2 n \sum_{j=1}^{L} \mu_{j}} \prod_{i=1}^{n}\left(x_{i}^{B} x_{i}^{C}\right)^{\frac{L-1}{2}} \\
& \times \sum_{\substack{1 \leq a_{1}<\cdots<a_{n} \leq L \\
1 \leq b_{1}<\cdots<b_{n} \leq L}} e^{\sum_{j=1}^{n} \mu_{a_{j}}+\mu_{b_{j}}}\left\langle 0\left|\prod_{1 \leq j \leq n}^{\longrightarrow} P_{a_{j}}^{+} \prod_{1 \leq j \leq n}^{\longrightarrow} P_{b_{j}}^{-}\right| 0\right\rangle,
\end{aligned}
$$

in the limit $x_{i}^{B, C} \rightarrow \infty$. The next step is to compute the quantity $\left\langle 0\left|\prod_{1 \leq j \leq n} P_{a_{j}}^{+} \prod_{1 \leq j \leq n} P_{b_{j}}^{-}\right| 0\right\rangle$ appearing in (D.10), and for that we notice that under the constraint $1 \leq a_{1}<\cdots<$ $a_{n} \leq L$ and $1 \leq b_{1}<\cdots<b_{n} \leq L$ the aforementioned quantity will contribute only when $b_{j}=a_{j}$. In that case we have $\left\langle 0\left|\prod_{1 \leq j \leq n} P_{a_{j}}^{+} \prod_{1 \leq j \leq n} P_{a_{j}}^{-}\right| 0\right\rangle=q^{n(n-1)}$ and the relation (D.10) then simplifies to

$$
S_{n} \sim \frac{\left(q-q^{-1}\right)^{2 n}}{2^{2 n L}} q^{n(L-n)}[n !]_{q^{2}}^{2} e^{-2 n \sum_{j=1}^{L} \mu_{j}} \sum_{1 \leq a_{1}<\cdots<a_{n} \leq L} e^{2 \sum_{j=1}^{n} \mu_{a_{j}}} \prod_{i=1}^{n}\left(x_{i}^{B} x_{i}^{C}\right)^{\frac{L-1}{2}} .
$$




\section{E Off-shell solution for the case $n=1$}

The relation (4.18) offers a way of building the solution of $(3.4,3.9)$ recursively. More precisely, the formula (4.18) establishes a relation between the scalar product $S_{n}$ and an auxiliary function $V$ consisting essentially of $S_{n-1}$ for a lattice of length $L-1$. Thus, since we are considering $n \leq L$, at the final step of this iteration procedure we shall need the solution of Eqs. $(\overline{3.4})$ and $(\overline{3.9})$ for the case $n=1$. In that case our system of functional equations explicitly read

$$
\begin{aligned}
& M_{0} S_{1}\left(\lambda_{1}^{C} \mid \lambda_{1}^{B}\right)+N_{1}^{(B)} S_{1}\left(\lambda_{1}^{C} \mid \lambda_{0}\right)+N_{1}^{(C)} S_{1}\left(\lambda_{0} \mid \lambda_{1}^{B}\right)=0 \\
& \widetilde{M}_{0} S_{1}\left(\lambda_{1}^{C} \mid \lambda_{1}^{B}\right)+\widetilde{N}_{1}^{(B)} S_{1}\left(\lambda_{1}^{C} \mid \lambda_{0}\right)+\widetilde{N}_{1}^{(C)} S_{1}\left(\lambda_{0} \mid \lambda_{1}^{B}\right)=0
\end{aligned}
$$

with coefficients

$$
\begin{array}{rlrl}
M_{0} & =\frac{c\left(\lambda_{0}-\lambda_{1}^{B}\right)}{b\left(\lambda_{0}-\lambda_{1}^{B}\right)} \frac{b\left(\lambda_{1}^{B}-\lambda_{1}^{C}\right)}{b\left(\lambda_{0}-\lambda_{1}^{C}\right)} \prod_{j=1}^{L} a\left(\lambda_{0}-\mu_{j}\right) & N_{1}^{(B)}=\frac{c\left(\lambda_{1}^{B}-\lambda_{0}\right)}{b\left(\lambda_{1}^{B}-\lambda_{0}\right)} \prod_{j=1}^{L} a\left(\lambda_{1}^{B}-\mu_{j}\right) \\
\widetilde{M}_{0}=\frac{c\left(\lambda_{0}-\lambda_{1}^{B}\right)}{b\left(\lambda_{0}-\lambda_{1}^{B}\right)} \frac{b\left(\lambda_{1}^{C}-\lambda_{1}^{B}\right)}{b\left(\lambda_{0}-\lambda_{1}^{C}\right)} \prod_{j=1}^{L} b\left(\lambda_{0}-\mu_{j}\right) & \widetilde{N}_{1}^{(B)}=\frac{c\left(\lambda_{0}-\lambda_{1}^{B}\right)}{b\left(\lambda_{0}-\lambda_{1}^{B}\right)} \prod_{j=1}^{L} b\left(\lambda_{1}^{B}-\mu_{j}\right) \\
N_{1}^{(C)}=\frac{c\left(\lambda_{0}-\lambda_{1}^{C}\right)}{b\left(\lambda_{0}-\lambda_{1}^{C}\right)} \prod_{j=1}^{L} a\left(\lambda_{1}^{C}-\mu_{j}\right) & \widetilde{N}_{1}^{(C)}=\frac{c\left(\lambda_{1}^{C}-\lambda_{0}\right)}{b\left(\lambda_{1}^{C}-\lambda_{0}\right)} \prod_{j=1}^{L} b\left(\lambda_{1}^{C}-\mu_{j}\right),
\end{array}
$$

and the solution of $(\overline{E .1})$ can be obtained as follows. By eliminating the term $S_{1}\left(\lambda_{0} \mid \lambda_{1}^{B}\right)$ from (E.1), we obtain an equation involving only the terms $S_{1}\left(\lambda_{1}^{C} \mid \lambda_{1}^{B}\right)$ and $S_{1}\left(\lambda_{1}^{C} \mid \lambda_{0}\right)$. More precisely, we are left with the identity

$$
\frac{b\left(\lambda_{1}^{B}-\lambda_{1}^{C}\right) S_{1}\left(\lambda_{1}^{C} \mid \lambda_{1}^{B}\right)}{\left[\prod_{j=1}^{L} \frac{a\left(\lambda_{1}^{B}-\mu_{j}\right)}{a\left(\lambda_{1}^{C}-\mu_{j}\right)}-\prod_{j=1}^{L} \frac{b\left(\lambda_{1}^{B}-\mu_{j}\right)}{b\left(\lambda_{1}^{C}-\mu_{j}\right)}\right]}=\frac{b\left(\lambda_{0}-\lambda_{1}^{C}\right) S_{1}\left(\lambda_{1}^{C} \mid \lambda_{0}\right)}{\left[\prod_{j=1}^{L} \frac{a\left(\lambda_{0}-\mu_{j}\right)}{a\left(\lambda_{1}^{C}-\mu_{j}\right)}-\prod_{j=1}^{L} \frac{b\left(\lambda_{0}-\mu_{j}\right)}{b\left(\lambda_{1}^{C}-\mu_{j}\right)}\right]},
$$

where the variable $\lambda_{1}^{C}$ now assumes the role of a parameter. The relation $(\bar{E} .3)$ is then readily solved by

$$
S_{1}\left(\lambda_{1}^{C} \mid \lambda_{1}^{B}\right)=\frac{F\left(\lambda_{1}^{C}\right)}{b\left(\lambda_{1}^{C}-\lambda_{1}^{B}\right)}\left[\prod_{j=1}^{L} \frac{a\left(\lambda_{1}^{B}-\mu_{j}\right)}{a\left(\lambda_{1}^{C}-\mu_{j}\right)}-\prod_{j=1}^{L} \frac{b\left(\lambda_{1}^{B}-\mu_{j}\right)}{b\left(\lambda_{1}^{C}-\mu_{j}\right)}\right],
$$

where $F$ is an arbitrary function. Next we substitute the expression (E.4) into the first Eq. of (E.1), and after eliminating an overall factor we are left with the relation

$$
\begin{aligned}
& F\left(\lambda_{1}^{C}\right)\left[\prod_{j=1}^{L} \frac{a\left(\lambda_{1}^{B}-\mu_{j}\right) b\left(\lambda_{0}-\mu_{j}\right)}{b\left(\lambda_{1}^{C}-\mu_{j}\right)}-\prod_{j=1}^{L} \frac{a\left(\lambda_{0}-\mu_{j}\right) b\left(\lambda_{1}^{B}-\mu_{j}\right)}{b\left(\lambda_{1}^{C}-\mu_{j}\right)}\right] \\
& -F\left(\lambda_{0}\right)\left[\prod_{j=1}^{L} \frac{a\left(\lambda_{1}^{B}-\mu_{j}\right) a\left(\lambda_{1}^{C}-\mu_{j}\right)}{a\left(\lambda_{0}-\mu_{j}\right)}-\prod_{j=1}^{L} \frac{a\left(\lambda_{1}^{C}-\mu_{j}\right) b\left(\lambda_{1}^{B}-\mu_{j}\right)}{b\left(\lambda_{0}-\mu_{j}\right)}\right]=0 .
\end{aligned}
$$


As far as the Eq. (E.5) is concerned, the variable $\lambda_{1}^{B}$ is merely a parameter which can be set at our convenience. In particular, for the specialisation $\lambda_{1}^{B}=\mu_{k}-\gamma$ the Eq. (E.5) simplifies to

$$
\frac{F\left(\lambda_{0}\right)}{\prod_{j=1}^{L} a\left(\lambda_{0}-\mu_{j}\right) b\left(\lambda_{0}-\mu_{j}\right)}=\frac{F\left(\lambda_{1}^{C}\right)}{\prod_{j=1}^{L} a\left(\lambda_{1}^{C}-\mu_{j}\right) b\left(\lambda_{1}^{C}-\mu_{j}\right)} .
$$

Eq. (E.6) can then be easily solved and we find

$$
F(\lambda)=\Omega \prod_{j=1}^{L} a\left(\lambda-\mu_{j}\right) b\left(\lambda-\mu_{j}\right)
$$

where $\Omega$ is a $\lambda$ independent parameter. Then the combination of (E.4) and (E.7) yields the expression

$$
S_{1}\left(\lambda_{1}^{C} \mid \lambda_{1}^{B}\right)=\frac{c\left(\lambda_{1}^{C}-\lambda_{1}^{B}\right)}{b\left(\lambda_{1}^{C}-\lambda_{1}^{B}\right)}\left[\prod_{j=1}^{L} a\left(\lambda_{1}^{B}-\mu_{j}\right) b\left(\lambda_{1}^{C}-\mu_{j}\right)-\prod_{j=1}^{L} a\left(\lambda_{1}^{C}-\mu_{j}\right) b\left(\lambda_{1}^{B}-\mu_{j}\right)\right]
$$

where the constant $\Omega$ has been fixed by the asymptotic behaviour (D.11).

\section{References}

[1] E. K. Sklyanin. Method of the inverse scattering problem and the nonlinear quantum Schrödinger equation. Sov. Phys. Dokl., 24:107, 1979.

[2] W. Heisenberg. Zur Theorie des Ferromagnetismus. Zeitschrift für Physik, 49(910):619-636, 1928.

[3] J. Hubbard. Electron correlations in narrow energy bands. Proc. R. Soc. Lond. A-Math. Phys. Sci., 276(1364):238, 1963.

[4] J.-S. Caux and J. Mossel. Remarks on the notion of quantum integrability. J. Stat. Mech.-Theory Exp., (02):P02023, 2011.

[5] S. Weigert. The problem of quantum integrability. Physica D, 56(1):107-119, 1992.

[6] L.D. Faddeev. What is complete integrability in quantum mechanics. Proceedings of the Symposium Henri Poincaré, October 2004.

[7] J. Clemente-Gallardo and G. Marmo. Towards a definition of quantum integrability. Int. J. Geom. Methods Mod. Phys., 6(1):129-172, 2009.

[8] V. E. Korepin, N. M. Bogoliubov, and A. G. Izergin. Quantum inverse scattering method and correlation functions. Cambridge University Press, 1993. 
[9] F. H. L Essler, H. Frahm, F. Göhmann, A. Klümper, and V. E. Korepin. The One-Dimensional Hubbard Model. Cambridge University Press, Cambridge, 2005.

[10] H. Bethe. Zur Theorie der Metalle I. Eigenwerte und Eigenfunktionen der Linearen Atomkette. Zeitschrift für Physik, (71):225-226, 1931.

[11] E. K. Sklyanin, L. A. Takhtadzhyan, and L. D. Faddeev. Quantum Inverse Problem Method .1. Theor. Math. Phys., 40(2):688-706, 1979.

[12] L. A. Takhtadzhyan and L. D. Faddeev. The quantum method of the inverse problem and the Heisenberg XZY model. Russ. Math. Surv., 11(34), 1979.

[13] V. O. Tarasov. Algebraic Bethe ansatz for the Izergin-Korepin $R$-matrix. Theor. Math. Phys., 76(2):793-803, 1988.

[14] M. J. Martins and P. B. Ramos. The quantum inverse scattering method for Hubbard-like models. Nucl. Phys. B, 522(3):413-470, 1998.

[15] W. Galleas and M. J. Martins. $R$-matrices and spectrum of vertex models based on superalgebras. Nucl. Phys. B, 699(3):455-486, 2004.

[16] C. S. Melo and M. J. Martins. Algebraic Bethe ansatz for $U(1)$ invariant integrable models: The method and general results. Nucl. Phys. B, 806(3):567-635, 2009.

[17] B. M. McCoy. The Baxter revolution. J. Stat. Phys., 102(3-4):375-384, 2001.

[18] R. J. Baxter. Eight vertex model in lattice statistics. Phys. Rev. Lett., 26:832, 1971.

[19] R. J. Baxter. Partition-function of 8-vertex lattice model. Ann. Phys., 70(1):193, 1972 .

[20] N. Y. Reshetikhin. The spectrum of the transfer-matrices connected with KacMoody algebras. Lett. Math. Phys., 14(3):235-246, 1987.

[21] Y. G. Stroganov. A new calculation method for partition functions in some lattice models. Phys. Lett. A, 74:116, 1979.

[22] W. Galleas. Functional relations from the Yang-Baxter algebra: Eigenvalues of the $X X Z$ model with non-diagonal twisted and open boundary conditions. Nucl. Phys. $B, 790(3): 524-542,2008$.

[23] V. E. Korepin. Calculation of norms of Bethe wave functions. Commun. Math. Phys., 86:391-418, 1982.

[24] M. Gaudin. La Fonction D'onde De Bethe. Masson, Paris, 1983.

[25] N. Kitanine, J. M. Maillet, and V. Terras. Form factors of the $X X Z$ Heisenberg spin- $\frac{1}{2}$ finite chain. Nucl. Phys. B, 554(3):647-678, 1999.

[26] W. Galleas. Functional relations for the six-vertex model with domain wall boundary conditions. J. Stat. Mech., (06):P06008, 2010. 
[27] W. Galleas. A new representation for the partition function of the six-vertex model with domain wall boundaries. J. Stat. Mech., (01):P01013, 2011.

[28] W. Galleas. Multiple integral representation for the trigonometric SOS model with domain wall boundaries. Nucl. Phys. B, 858(1):117-141, 2012, math-ph/1111.6683.

[29] W. Galleas. Refined functional relations for the elliptic SOS model. Nucl. Phys. B, 867:855-871, 2013.

[30] R. J. Baxter. Exactly Solved Models in Statistical Mechanics. Dover Publications, Inc., Mineola, New York, 2007.

[31] E. H. Lieb. Residual entropy of square lattice. Phys. Rev., 162(1):162, 1967.

[32] B. M. McCoy and T. T. Wu. Hydrogen-bonded crystals and anistropic Heisenberg chain. Nuovo Cimento B, 55(1):B7-\&, 1968.

[33] B. Sutherland. 2-dimensional hydrogen bonded crystals without ice rule. J. Math. Phys., 11(11):3183-\&, 1970.

[34] H. J. de Vega. Families of commuting transfer matrices and integrable models with disorder. Nucl. Phys. B, 240(4):495-513, 1984.

[35] N. A. Slavnov. Calculation of scalar products of wave functions and form factors in the framework of the algebraic Bethe ansatz. Theor. Math. Phys., 79(2):502-508, 1989 .

[36] J. de Gier, W. Galleas, and M. Sorrell. Multiple integral formula for the off-shell six vertex scalar product. 2011, hep-th/1111.3712.

[37] M. Wheeler. Scalar products in generalized models with $S U(3)$-symmetry. 2012, arXiv: 1204.2089 .

[38] S. Belliard, S. Pakuliak, E. Ragoucy, and N. A. Slavnov. Highest coefficient of scalar products in SU(3)-invariant integrable models. J. Stat. Mech., (09):P09003, 2012.

[39] S. Belliard, S. Pakuliak, E. Ragoucy, and N. A. Slavnov. The algebraic Bethe ansatz for scalar products in SU(3)-invariant integrable models. J. Stat. Mech., (10):P10017, 2012.

[40] S. Belliard, S. Pakuliak, E. Ragoucy, and N. A. Slavnov. Bethe vectors of SU(3)invariant integrable models. 2012, arXiv: 1210.0768.

[41] S. Belliard, S. Pakuliak, E. Ragoucy, and N. A. Slavnov. Form factors in SU(3)invariant integrable models. 2012, arXiv: 1211.3968. 